\title{
Preparation and antitumor evaluation of self-
} assembling oleanolic acid-loaded Pluronic PI05/D$\alpha$-tocopheryl polyethylene glycol succinate mixed micelles for non-small-cell lung cancer treatment

\author{
This article was published in the following Dove Press journal: \\ International Journal of Nanomedicine \\ 28 November 2016 \\ Number of times this article has been viewed
}

\author{
Hao Wu, ${ }^{1-3}$ Qingxiang \\ Zhong, ${ }^{1,2}$ Rongling Zhong, ${ }^{4}$ \\ Houcai Huang, ${ }^{4}$ Zhi Xia, ${ }^{4}$ \\ Zhongcheng Ke, ${ }^{1,5}$ Zhenhai \\ Zhang,' Jie Song, ${ }^{1,2}$ Xiaobin \\ $\mathrm{Jia}^{1-3}$
}

'Affiliated Hospital of Integrated Traditional Chinese and Western Medicine, Nanjing University of Chinese Medicine, ${ }^{2}$ Key Laboratory of New Drug Delivery System of Chinese Materia Medica, Jiangsu Province Academy of Chinese Medicine, Nanjing, Jiangsu, ${ }^{3}$ College of Pharmacy, Anhui University of Chinese Medicine, Hefei, Anhui, ${ }^{4}$ Laboratory Animal Center, Jiangsu Province Academy of Chinese Medicine, Nanjing, Jiangsu, ${ }^{5}$ College of Chemistry and Chemical Engineering, Huangshan University, Huangshan, Anhui, People's Republic of China

Correspondence: Jie Song Key Laboratory of New Drug Delivery System of Chinese Material Medica, Jiangsu Province Academy of Chinese Medicine, 100 Shizi Street, Hongshan Road, Jiangsu, Nanjing 21 0028, People's Republic of China

Tel +862585637960

Email momo198420@hotmail.com

Xiaobin Jia

Affiliated Hospital of Integrated

Traditional Chinese and Western

Medicine, Nanjing University of Chinese

Medicine, 100 Shizi Street, Hongshan

Road, Jiangsu, Nanjing 210028, People's

Republic of China

Tel +862585637809

Fax +862585502829

Email jiaxiaobin2015@163.com

\begin{abstract}
Oleanolic acid (OA) is a triterpenoid found in various fruits and vegetables and used in traditional Chinese medicine. OA plays a crucial role in the treatment of several cancers, but poor water solubility, low permeability, and significant efflux have limited its widespread clinical use. Vitamin E-D- $\alpha$-tocopheryl polyethylene glycol succinate (vitamin E-TPGS) and Pluronic P105 were used to improve the solubility and permeability and to decrease the efflux of OA. OA-loaded mixed micelles were prepared by ethanol thin-film hydration. The physicochemical properties of the micelles, including zeta potential, morphology, particle size, solubility, drug loading, and drug entrapment efficiency were characterized. OA release from micelles was slower than that from the free drug system. OA uptake by A549 non-small-cell lung cancer (NSCLC) cells was enhanced by the micelles. A tumor model was established by injecting A549 cells into nude mice. In vivo imaging showed that OA-micelles could accumulate in the tumors of nude mice. Additionally, smaller tumor size and increased expression of pro-apoptotic proteins were observed in OA-micelle-treated mice, indicating that OA-micelles are more effective than free OA in treating cancer. In vitro experiments were performed using two NSCLC cell lines (A549 and PC-9). Cytotoxicity evaluations showed that the half-maximal inhibitory concentrations of free OA and OA-micelles were $36.8 \pm 4.8$ and $20.9 \pm 3.7 \mu \mathrm{M}$, respectively, in A549 cells and $82.7 \pm 7.8$ and $56.7 \pm 4.7 \mu \mathrm{M}$, respectively, in PC-9 cells. Apoptosis assays revealed that the apoptotic rate of OA-micelle-treated A549 and PC-9 cells was higher than that of cells treated with the same concentration of free OA. Wound healing and transwell assays showed that migration and invasion were significantly suppressed in OA-micelle-treated cells. Immunofluorescence and Western blot analyses confirmed that the epithelial-mesenchymal transition was reversed in OA-micelle-treated cells. Mixed micelles are a promising nano-drug delivery system for lung cancer treatment.
\end{abstract}

Keywords: oleanolic acid, Pluronic P105, vitamin E-TPGS, polymer-drug conjugate, NSCLC

\section{Introduction}

Lung cancer is one of the leading causes of mortality in the world and has a rapidly increasing incidence. ${ }^{1}$ The symptoms of early-stage lung cancer are not obvious, and clinical diagnosis is often not made until an advanced stage. Therefore, there is a high metastasis and mortality rate in patients. Current chemotherapeutic strategies lack broad specificity and efficacy. Although surgical resection is an alternative treatment for lung cancer, prognoses are poor and the survival rate is extremely low. Therefore, lung cancer remains a serious threat to both health and quality of life. ${ }^{2}$ 
Non-small-cell lung cancer (NSCLC) is the most common lung cancer type and accounts for $85 \%-90 \%$ of lung cancer. Thus, it is important to research and develop an effective therapeutic method to treat NSCLC. In addition to current chemotherapeutic strategies and surgical resection, some natural products have emerged as effective anticancer agents. Oleanolic acid (OA; Figure 1) is a natural product with potential antitumor activity and can induce apoptosis in many tumor cell lines, including those of the breast, lung, and liver..$^{3-5}$ Unfortunately, the poor water solubility and low permeability of OA have limited its use. ${ }^{6}$ Therefore, it is necessary to select an appropriate nano-drug delivery system to increase the solubility of OA and to reduce the oral first pass effect and improve bioavailability.

Mixed micelles are emerging as new drug delivery vehicles. Recently, mixed micelles have been used to improve the solubility and bioavailability of hydrophobic drugs. Therefore, mixed micelles could be used to overcome the current limitations of OA use in the treatment of cancer. Vitamin E-D- $\alpha$-tocopheryl polyethylene glycol succinate (vitamin E-TPGS) and Pluronic P105 (P105) were used as surfactants to improve the solubility and permeability and to decrease the efflux of OA. TPGS is an efflux inhibitor with a water-soluble amphiphilic molecular structure. ${ }^{7}$ The ethylene oxide-propylene oxide (EO-PO) triblock copolymers have been extensively investigated. $\mathrm{P} 105$ is a $\mathrm{PEO}_{37}-\mathrm{PPO}_{56}-\mathrm{PEO}_{37}$ triblock copolymer with a molecular weight of 6,500 $\mathrm{Da}$ and the potent ability to sensitize multidrug-resistant tumors and the capacity to encapsulate hydrophobic drugs. ${ }^{8,9} \mathrm{OA}$-loaded P105/TPGS mixed micelles (OA-micelles) were prepared using a thin-film hydration method and possessed the aforementioned advantages. Thus, nanotechnological and nanoscale materials exhibit a considerable potential for increasing solubility and enhancing absorption of drugs. Apoptosis plays a central role in antitumor therapy. The

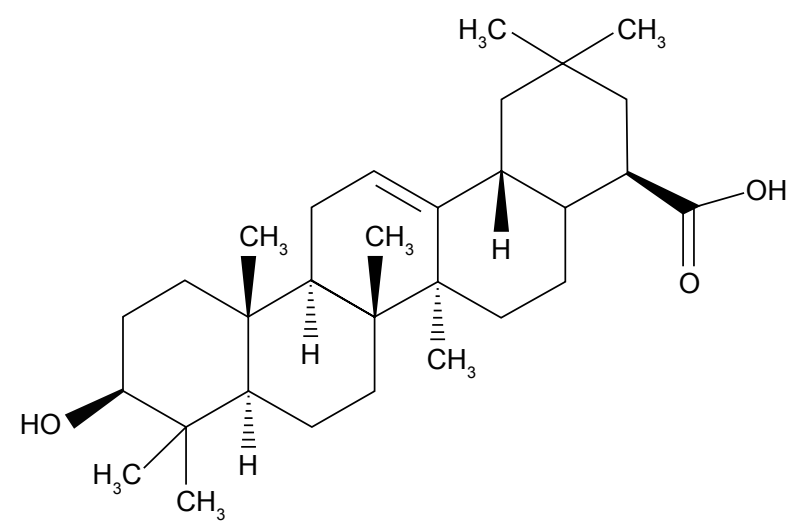

Figure I Chemical structure of oleanolic acid. apoptosis-inducing effect of free OA and OA-micelles in two NSCLC cell lines, A549 and PC-9, was compared. The results show that OA-micelles significantly reduce tumor size in vivo and inhibit invasion, migration, and the epithelialmesenchymal transition (EMT) in vitro. Overall, the TPGS and P105 mixed micelle is an appropriate drug delivery system for OA to treat NSCLC.

\section{Experiments Materials}

OA (purity $>98 \%$ ) was purchased from YuanYe Bio-Technology Co., Ltd (Shanghai, People's Republic of China). Pluronic P105 was purchased from Sigma-Aldrich (St Louis, MO, USA). TPGS was purchased from Aladdin Biochemical Technology Co., Ltd (Shanghai, People's Republic of China). Milli-Q water (Millipore, Bedford, MA, USA) was used throughout the research. Ethanol was purchased from Nanjing Chemical Reagent Co., Ltd (Nanjing, People's Republic of China). All reagents were of analytical grade. Dulbecco's Modified Eagle's Medium (DMEM) and fetal bovine serum (FBS) were purchased from Life Technologies AG (Solothurn, Switzerland). Coumarin 6 (C6), 4',6-diamidino-2-phenylindole (DAPI), 1,1'-dioctadecyl$3,3,3^{\prime}, 3^{\prime}$-tetramethyl indotricarbocyanine iodide (DiR) dimethyl sulfoxide (DMSO), and 3-(4,5-dimethylthiazol-2yl)-2,5-diphenyltetrazolium bromide (MTT) were purchased from Sigma-Aldrich. Ethanol, phosphate-buffered saline (PBS), and trypsin-ethylene diamine tetraacetic acid (EDTA) were supplied by Nanjing Jiancheng Bioengineering Institute (Nanjing, People's Republic of China).

\section{Animals}

Male nude mice (4 weeks old, $20 \pm 2 \mathrm{~g}$ ) were purchased from the SLAC Lab Animal Center of Shanghai (Shanghai, People's Republic of China). All animals were maintained under specific pathogen-free conditions at $20^{\circ} \mathrm{C}-24^{\circ} \mathrm{C}, 50 \%-70 \%$ humidity, and $12 \mathrm{~h} / 12 \mathrm{~h}$ light-dark cycle in the Animal Laboratory Center of Jiangsu Research Institute of Traditional Chinese Medicine. All animal experiments were reviewed and approved by the Animal Care and Use Committee of the Animal Laboratory Center of Jiangsu Research Institute of Traditional Chinese Medicine and adhered to the guidelines of the Guide for the Care and Use of Laboratory Animals.

The nude mice tumor model was developed by subcutaneously injecting $200 \mu \mathrm{L}$ of A549 cell suspension at $\sim 2 \times 10^{6}$ cells/mouse into the right flank of each nude mouse. ${ }^{10}$ When the tumor volume reached $100-200 \mathrm{~mm}^{3}$, mice were randomly divided into four groups with six mice in each group. 
Two groups of mice were used as micelles and free OA treatment groups, another group was used as the positive control group, and the last group was used as the blank group.

\section{Preparation and characterization of OA-loaded mixed micelles}

\section{Preparation of OA-loaded mixed micelles}

OA-loaded mixed micelles were prepared by the solvent evaporation method. Then, $120 \mathrm{mg}$ of TPGS and $80 \mathrm{mg}$ of P105 were dissolved in $8 \mathrm{~mL}$ of acetone. OA (7 mg) was slowly added to the above solution. The solution was evaporated by a vacuum of $-0.1 \mathrm{PMa}$ at $50^{\circ} \mathrm{C}$ until the ethanol had evaporated. Next, $5 \mathrm{~mL}$ of deionized water was added to the bottle and shaken until a clear solution formed. The resultant mixture was filtered through a $0.22 \mu \mathrm{m}$ polyethersulfone syringe filter into a sterile tube, and micelles were successfully prepared. ${ }^{11}$

\section{Characterization of OA-loaded mixed micelles}

Particle size, polydispersity, and zeta potential of OA mixed micelles were determined by dynamic light scattering using a Malvern system (Malvern ZE SA, Worcestershire, UK); the micelles were equilibrated at $25^{\circ} \mathrm{C}$ for $5 \mathrm{~min}$ before testing. ${ }^{12}$ The results are shown as the average of three experiments.

\section{Morphology characterization}

The morphology of OA-micelles was observed with a transmission electron microscope. Diluted OA-micelles were placed on a copper grid and dried under vacuum pressure. Dried micelles were stained with 3\% phosphotungstic acid for $30 \mathrm{~s}$, and the micelle morphology was examined. ${ }^{13}$

\section{Determination of encapsulation efficiency and drug loading}

To determine the encapsulation efficiency (EE) of OA in micelles, drug-loaded micelles were dissolved in acetonitrile and the solution was filtered with a $0.45 \mu \mathrm{m}$ syringe filter. ${ }^{14}$ The OA content was analyzed by high-performance liquid chromatography (HPLC). The mobile phase was acetonitrile and double-distilled water (9:1, v:v) at a flow rate of $1 \mathrm{~mL} / \mathrm{min}$. The analysis was performed using a C18 column, and the temperature was maintained at $30^{\circ} \mathrm{C} .{ }^{15}$ The $\mathrm{EE}(\%)$ and drug loading (DL) (\%) of the OA-micelles were determined by the following equation:

$$
\mathrm{EE} \%=\frac{\mathrm{OA} \text { weight measured in nanomicelles }}{\mathrm{OA} \text { weight added in nanomicelles }} \times 100 \%
$$

$$
\mathrm{DL} \%=\frac{\text { The weight of OA }}{\text { The weight of OA and polymer }} \times 100 \%
$$

\section{Cell line and culture}

The A549 human lung cancer cell line was purchased from KeyGen Biotech Co., Ltd. (Nanjing, People's Republic of China). A549 cells were cultured in high-glucose DMEM, supplemented with $10 \% \mathrm{FBS}$, at $37^{\circ} \mathrm{C}$ in a $5 \% \mathrm{CO}_{2}$ atmosphere. ${ }^{16}$ The medium was changed every other day. When the cells reached $90 \%$ confluence, they were trypsinized with $0.25 \%$ trypsin-EDTA and subcultured. ${ }^{17}$

\section{In vitro drug release}

In vitro release of $\mathrm{OA}$ from micelles was investigated in a simulated blood $\mathrm{pH}$ environment ( $\mathrm{pH}$ 7.4). The experiment was performed as previously described. ${ }^{18}$ Briefly, $1 \mathrm{~mL}$ of OA-micelles (1.5 mg OA equivalent) was diluted with $9 \mathrm{~mL}$ PBS to a total volume of $10 \mathrm{~mL} ; 1 \mathrm{~mL}$ of this micelle suspension was used to determine the initial OA concentration, and the remaining $9 \mathrm{~mL}$ was poured into a dialysis bag (3,500 Da). The dialysis bag was sealed and immersed in $50 \mathrm{~mL}$ of PBS ( $\mathrm{pH} \mathrm{7.4)} \mathrm{containing} 0.2 \%$ Tween 80 to maintain sink conditions. The experiment was performed in triplicate at $37^{\circ} \mathrm{C}$ with shaking at $100 \mathrm{rpm}$ for $96 \mathrm{~h}$. Samples of $1 \mathrm{~mL}$ each were withdrawn at preset time intervals $(0,1,2$, $4,8,12,24,36,48,60,72,84$, and $96 \mathrm{~h}$ ) and replaced with the same volume of PBS containing $0.05 \%$ Tween 80 each time. The samples were analyzed by HPLC.

\section{Targeting test of micelles}

Male nude mice were subcutaneously injected in the right flank with $5 \times 10^{6}$ A549 cells. When the mean tumor diameter reached $\sim 6 \mathrm{~mm}$, mice were injected intravenously with micelles containing a near-infrared fluorescence probe, DiR. Each nude mouse was injected with $0.2 \mathrm{~mL}$ of $5 \mu \mathrm{g} / \mathrm{mL} \mathrm{DiR}$. The fluorescence distribution in nude mice was detected at $1,4,8,24$, and $48 \mathrm{~h}$ after injection. ${ }^{19}$

\section{In vitro cellular uptake}

In order to determine the affinity between NSCLC cells and micelles, the following experiment was performed. Cells in the logarithmic growth phase were seeded in 24-well plates at a density of $5 \times 10^{3}$ cells/well and cultured for $24 \mathrm{~h}$. C6 (10 mg), TPGS (120 mg), and P105 (80 mg) were dissolved in ethanol $(5 \mathrm{~mL})$ and dialyzed against three changes of water for $6 \mathrm{~h}$. The unentrapped C6 was removed by filtration through a $0.45 \mu \mathrm{m}$ membrane, and the C6-loaded 
micelles (C6-micelles) were obtained. ${ }^{20}$ C6-micelles were diluted with DMEM to a concentration of $10 \mu \mathrm{M}$ and added to the plates. The cells were subsequently cultured at $37^{\circ} \mathrm{C}$ for $2 \mathrm{~h}$ following which the medium was promptly discarded, and cold PBS was added to the plates to terminate cellular uptake. The cells were washed three times and fixed with anhydrous ethanol at $4^{\circ} \mathrm{C}$ for $30 \mathrm{~min}$ and incubated with DAPI $(5 \mu \mathrm{g}$ / $\mathrm{mL}$ ) for $10 \mathrm{~min}^{21}$ The cells were washed with PBS and observed under a fluorescence microscope (Olympus Corp., Tokyo, Japan).

\section{Anticancer study and mechanism study of OA-micelles}

\section{Anticancer study of OA-micelles}

Male nude mice (18-20 g) were housed six mice per group in the animal research laboratory in standard conditions in topfiltered cages. This study and experimental protocols were approved by the Institutional Animal Care and Use Committee (IACUC) of Jiangsu Province Academy of Chinese Medicine. Mice were injected subcutaneously with $200 \mu \mathrm{L}$ of A549 cell suspension $\left(1 \times 10^{6}\right.$ cells) into the right flank. ${ }^{22}$ When the mean tumor diameter reached $\sim 6 \mathrm{~mm}$, the tumorbearing mice were randomly divided into four groups. Each of the four groups received one of the following treatments by tail vein injection daily for 7 days: normal saline, OA $(0.1 \mathrm{~g} / \mathrm{kg})$, OA-micelles $(0.1 \mathrm{~g} / \mathrm{kg})$, or cisplatin. All mice were sacrificed by cervical dislocation at day 14 , and the tumors were immediately harvested and measured. Tumor volume was calculated using the following formula:

$$
V=a^{2} \times b / 2
$$

where $a$ is the smallest diameter and $b$ is the largest diameter. ${ }^{23}$

Tumors were fixed in 4\% paraformaldehyde for immunohistochemical analysis.

\section{Immunohistochemical analysis}

Tumor tissues fixed in 4\% paraformaldehyde were processed and trimmed, embedded in paraffin, and sectioned to a thickness of $\sim 10 \mu \mathrm{m}$. Sections were dewaxed and rehydrated with freshly distilled water. Sections were stained with rabbit antihuman polyclonal caspase-3, Bax, and Bcl-2 antibodies (Abcam, Cambridge, MA, USA). Sections were washed in PBS, incubated at room temperature for $10 \mathrm{~min}$, and stained with diaminobenzidine for $10 \mathrm{~min}$ and washed for $5 \mathrm{~min}$. Samples were stained with hematoxylin. Samples were dehydrated and mounted for microscopic examination. ${ }^{2}$

\section{In vitro activity of OA-micelles}

In vitro cytotoxicity study

In vitro cytotoxicity was determined by MTT assay. A549 cells were seeded in 96-well plates at 5,000 cells/well in $100 \mu \mathrm{L}$ of high-glucose DMEM medium containing 10\% FBS. Cells were incubated at $37^{\circ} \mathrm{C}$ with $5 \% \mathrm{CO}_{2}$ and $95 \%$ relative humidity for $24 \mathrm{~h}$. The medium was removed and replaced with OA, OA-micelles, and blank micelles at various concentrations. The cells were subjected to the MTT assay after $24 \mathrm{~h}$. The results at $570 \mathrm{~nm}$ were presented to assess cell inhibitory rate and inhibitory concentration values. ${ }^{24}$

\section{Cell apoptosis assay}

Apoptosis induction assays were performed with A549 and PC-9 cells treated with blank micelles, OA, and OAmicelles. First, Annexin V-fluorescein isothiocyanate (FITC)/propidium iodide (PI) stain was used to quantify apoptosis. Cells were plated on 6-well plates at a density of $1 \times 10^{5}$ cells/well and grown for $24 \mathrm{~h}$. After $24 \mathrm{~h}$, the medium was removed and the cells were exposed to medium containing blank micelles, OA, or OA-micelles. The cells were collected using pancreatin with no EDTA and stained with the Annexin V-FITC/PI Detection Kit as per instructions. Cells were analyzed using flow cytometry. ${ }^{25}$

\section{In vitro migration and invasion assays}

Wound healing assays were performed using monolayer A549 and PC-9 cells. Monolayer A549 and PC-9 cells were wounded by scratching with $200 \mu \mathrm{L}$ pipette tips and washed with prewarmed PBS to eliminate non-adhered cells. Cells were incubated for $24 \mathrm{~h}$, and then fresh serum-free DMEM containing blank micelles, free OA, or OA-micelles was added. The blank DMEM served as control. ${ }^{26}$ After $24 \mathrm{~h}$, images were taken using an OLYMPUS digital camera with an inverted microscope.

The transwell invasion assay was performed as previously described. ${ }^{27}$ Cells were treated with free OA or OA-micelles for $24 \mathrm{~h}$. The bottom chambers were filled with DMEM with $10 \%$ FBS. The top Matrigel-coated chambers were seeded with DMEM containing $1 \times 10^{5}$ A549 or PC-9 cells. Cells were allowed to migrate for $24 \mathrm{~h}$. Non-migrated cells were scraped off, and the migrated cells were fixed with methanol and stained with $0.05 \%$ crystal violet. Migrated cells were photographed with a light microscope and quantified by manual counting. The percentage of migrated cells inhibited by OA-micelles, relative to the DMEM-treated well, was calculated.

\section{Western blot}

A549 cells treated with free OA, OA-micelles, or the DMEM (control) were washed with PBS and lysed with RIPA lysis 
buffer (62.5 mM Tris [pH 6.8], 1\% sodium dodecyl sulfate [SDS], 10\% glycerol, and 5\% $\beta$-mercaptoethanol). ${ }^{28,29}$ The lysates were boiled for $5 \mathrm{~min}$. Equal amounts of protein were separated by SDS-polyacrylamide gel electrophoresis and transferred to Immobilon membranes (Millipore) for each treatment. Membranes were blocked with 4\% skim milk for $1 \mathrm{~h}$. Membranes were incubated with primary antibodies (1:500 dilution and washed three times with Tris-buffered saline Tween-20 [TBST]). Membranes were incubated with the corresponding secondary antibodies for $1 \mathrm{~h} .{ }^{30-32}$ Visualization of the protein bands was achieved using enhanced chemiluminescence protein bands. G:BOX chemiXR5 was used for imaging. Protein bands were quantified by densitometric analysis using the Gel-pro Analyzer.

\section{Immunofluorescence analysis}

The immunofluorescence analysis was utilized to determine the alteration of E-cadherin and $\mathrm{N}$-cadherin as previously described. A549 and PC-9 cells were seeded on 6-well plates and cultured overnight, after which cells were treated with free OA and OA-micelles. After another $24 \mathrm{~h}$ incubation, each sample was rinsed three times with PBS for 5 min and fixed with $4 \%$ polyoxymethylene before being treated with $0.5 \%$ Triton X-100. Cells were immunostained with primary antibody at $4^{\circ} \mathrm{C}$ overnight before being blocked with $5 \%$ bovine serum albumin for $1 \mathrm{~h}$. The coverslips were incubated with DAPI for $10 \mathrm{~min}$ at room temperature in a darkroom after being rinsed three times with PBS. Finally, the coverslips were resuspended in PBS for detection, and the polymerization of tubulin was imaged using an IN Cell Analyser. ${ }^{33}$

\section{Statistical analysis}

Data are presented as the mean \pm standard deviation. The statistical significance of treatment outcomes was assessed using Student's $t$-test (two-tailed); $P$-values $<0.05$ were considered statistically significant in all analyses.

\section{Results \\ Preparation and characterization of the OA-micelles}

Highly soluble OA-micelles were developed by single-step solvent evaporation method as previously reported. ${ }^{34}$ TPGS and P105 were dissolved in a ethanol and then combined with OA powder to evaporate in a rotary evaporator. During this process, OA and TPGS/P105 self-assembled into micelles, which encapsulated OA in the core part. Meanwhile, compared with the free OA, the micelles' formation had a significant inhibitory effect on the efflux (Figure 2$).{ }^{35}$

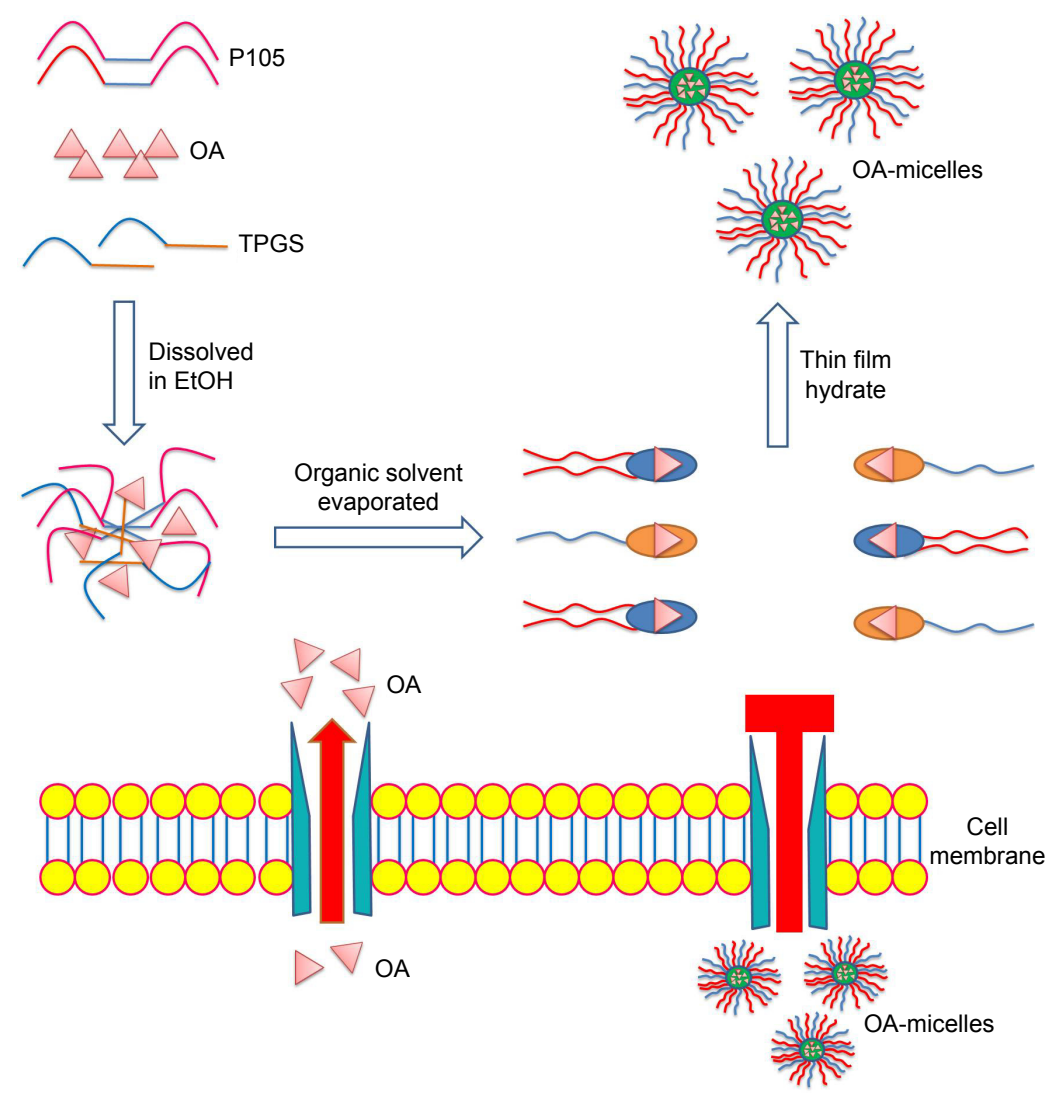

Figure 2 Preparation scheme of OA-micelles via the self-assembly method and different phenomena between OA-micelles and free OA in the cell. Abbreviations: EtOh, ethyl alcohol; OA, oleanolic acid; PI05, Pluronic PI05; TPGS, D- $\alpha$-tocopheryl polyethylene glycol succinate. 

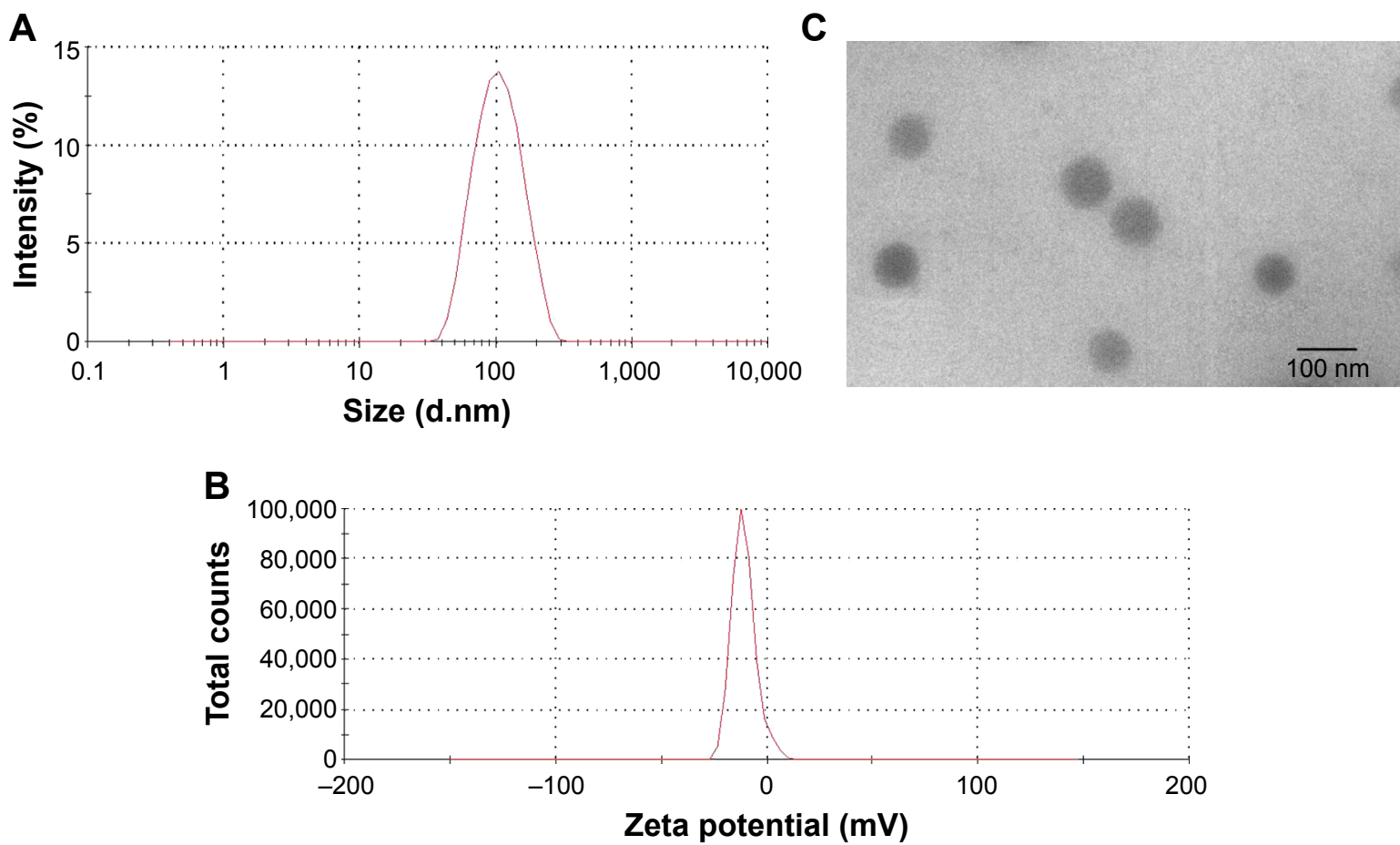

Figure 3 Characterization of OA-micelles.

Notes: (A) Size distribution spectrum of OA-micelles; (B) zeta potential spectrum of OA-micelles; and (C) transmission electron micrograph of OA-micelles (bar $100 \mathrm{~nm}$ ).

Abbreviation: OA, oleanolic acid.

The size distribution, zeta potential, and transmission electron micrograph are shown in Figure 3A. The size distribution spectrum showed that the OA-micelles were monodispersed and had a narrow particle size distribution (polydispersity index $=0.13 \pm 0.03$ ). The OA-micelles had an average diameter of $95.7 \pm 3.6 \mathrm{~nm}$ in aqueous phase and a DL of $3.5 \%$ and an EE of $93.6 \% \pm 0.05 \%$. The zeta potential of OA-micelles was $-8.6 \pm 0.4 \mathrm{mV}$ (Figure 3B). Spherical OA-micelles in the drying phase measured $\sim 80-90 \mathrm{~nm}$ under electron microscope (Figure 3C). The dehydration and shrinkage of micelles during sample processing may result in smaller diameters than those obtained by the dynamic light scattering technique. ${ }^{36}$

\section{In vitro drug release}

PBS ( $\mathrm{pH}$ 7.4) was chosen as a release environment to simulate blood $\mathrm{pH}$ conditions. OA-micelles and free $\mathrm{OA}$ were incubated in PBS containing $0.1 \%$ Tween 80 at $37^{\circ} \mathrm{C}$ and the OA concentration was determined at several time points. OA was released from the micelles at a much slower speed than from free OA (Figure 4). Approximately $80 \%$ of OA had been released from the dialysis bag containing free $\mathrm{OA}$ at $24 \mathrm{~h}$. Meanwhile, only $\sim 40 \%$ was released from the dialysis bag containing OA-micelles at the same time point (Figure 4). The reason for this phenomenon is that the OA contained within micelles is protected by the micelle core, making it much more stable than free OA. Rapid release of water-soluble drugs may induce drug precipitation or their binding to plasma proteins in vivo, effectively resulting in drug dilution and possibly leading to drug inactivation. ${ }^{37}$ Therefore, the slower release of OA from micelles can be an advantage for the antitumor effect of OA in vivo.

\section{Tumor targeting}

Nanoparticle drug delivery systems will facilitate tumor cell uptake. However, the maintenance time of drug accumulation

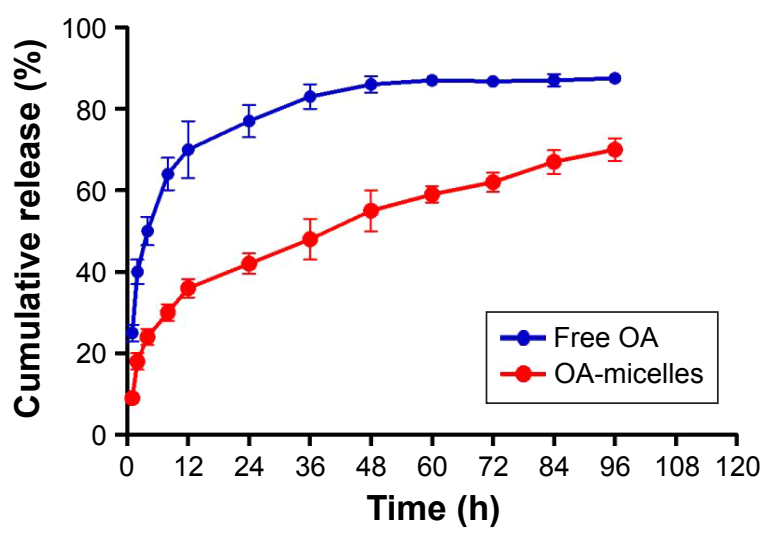

Figure 4 Cumulative release of $\mathrm{OA}$ from the OA-micelles and free OA in PBS (pH 7.4).

Abbreviations: OA, oleanolic acid; PBS, phosphate-buffered saline. 
at the tumor site is another meaningful consideration for clinical cancer therapeutics. DiR has been developed as a popular fluorescent dye with strong absorption in the nearinfrared region and is almost nontoxic at low concentration, making it suitable for in vivo fluorescence imaging. ${ }^{38,39}$ After intravenous injection, DiR-loaded micelles are observed in A549 tumor xenografts. Mice treated with DiR-micelles showed a certain time-dependent increase in the fluorescence signals, especially at $24 \mathrm{~h}$ after injection (Figure 5). These results suggest that the mixed OA-micelles probably had a targeting efficiency.

\section{Cellular uptake}

C6 is a fluorescent dye widely used in cellular uptake experiments as a substitute for hydrophobic drugs. In this study, C6-micelles were prepared and used in A549 and PC-9 cells for uptake experiments, and cell nuclei were stained blue by DAPI (Figure 6A and B). The green fluorescence signal in the C6-micelles group was significantly greater than that of the free $\mathrm{C} 6$ group, indicating that the micelles facilitated the uptake of C6 by A549 and PC-9 cells (Figure 6C). It can be extrapolated that the uptake of OA will also be greatly enhanced by the micelle delivery system.

\section{In vivo antitumor efficacy}

Nude mice inoculated with A549 cells were used to evaluate the antitumor efficacy of OA-micelles in vivo. Representative pictures of tumors from each group are shown in Figure 7A. Tumor volume of the control group increased significantly, while the growth in the cisplatin group and the OA-micelles group was greatly inhibited (Figure 7B). The average tumor volume of the group treated with free OA was close to that of the vehicle group, and the tumor volume of the OA-micelles group was significantly smaller than that of both the vehicle group $(P<0.01)$ and the free OA group $(P<0.05)$. Tumors were removed and weighed after the last injection. The average weight of tumors from the OA-micelles group mice was $1.42 \pm 0.16 \mathrm{~g}$, and the average weights of tumors from the free OA group and the vehicle group mice were $2.23 \pm 0.26$ and $3.87 \pm 0.29 \mathrm{~g}$, respectively. The tumor weight of the OA-micelle-treated group was significantly lower than that of the vehicle group $(P<0.01)$ and the free OA group $(P<0.05)$ (Figure 7C). This indicates that the $\mathrm{OA}$ in the micelle preparations had a significant antitumor effect. The same conclusion through the tumor volume and the tumor weight experiments was arrived at. There was no obvious body weight loss in mice after intravenous administration of OA-micelles and free OA (Figure 7D). However, the body weight of mice in the group treated with cisplatin was significantly reduced $(P<0.01)$ compared with other groups. Therefore, OA-micelles appear to be a safe and efficient antitumor treatment in tumor-bearing mice.

The relative levels of protein expression in the tumors of mice receiving different treatments were assessed using immunohistochemical staining. The average optical density indicative of $\mathrm{Bcl}-2$ expression was lower in the OA-micelles group than in the vehicle $(P<0.01)$ and free OA groups $(P<0.05)$ (Figure 8$)$. Bax and Caspase-3 proteins were more highly expressed in the OA-micelles group than in the vehicle or free OA groups. Additionally, the differences between the levels of Bax and Caspase- 3 expression in the OA-micelles and free OA groups were statistically significant $(P<0.05)$. Taken together, these results show that treatment with OA-micelles enhanced the $\mathrm{Bax} / \mathrm{Bcl}-2$ ratio and increased Caspase-3 expression, accelerating apoptosis in the tumors.

\section{Cell cytotoxicity and apoptosis-inducing effects of OA-micelles in NSCLC}

The NSCLC cytotoxicity of OA-micelles, compared with free OA, was studied in A549 and PC-9 cells. MTT assays showed
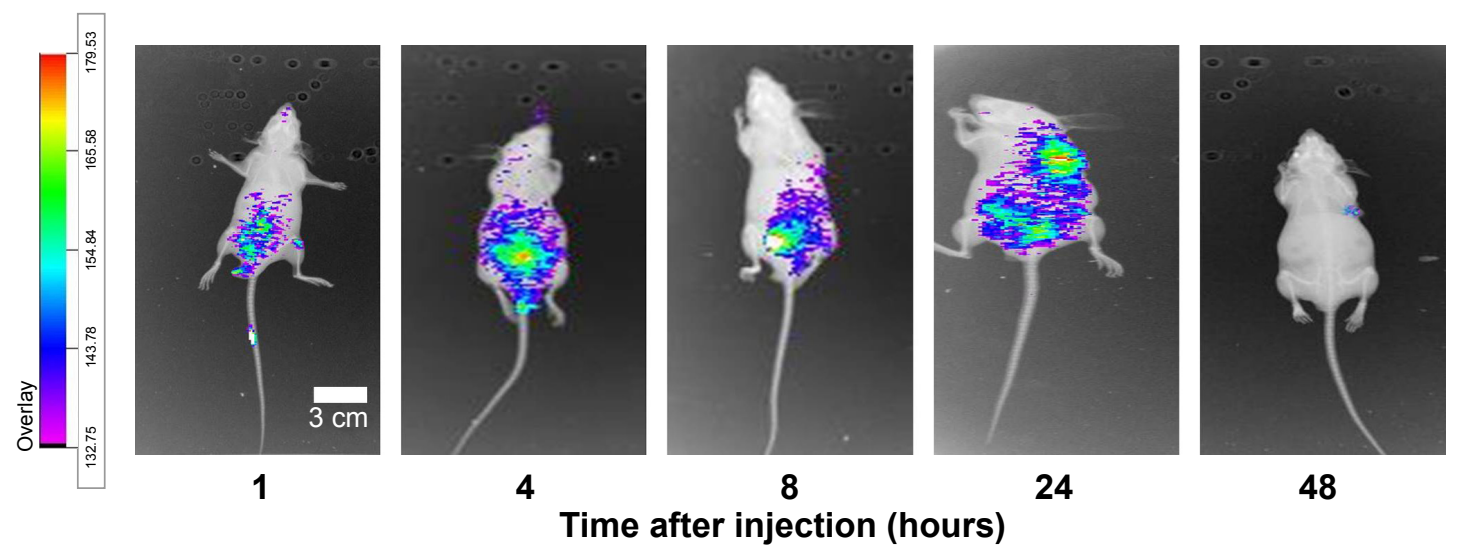

Figure 5 In vivo images of tumor-bearing mice after intravenous injection of DiR-micelles $(n=3)$. Abbreviation: DiR, I, I'-dioctadecyl-3,3,3',3'-tetramethyl indotricarbocyanine iodide. 

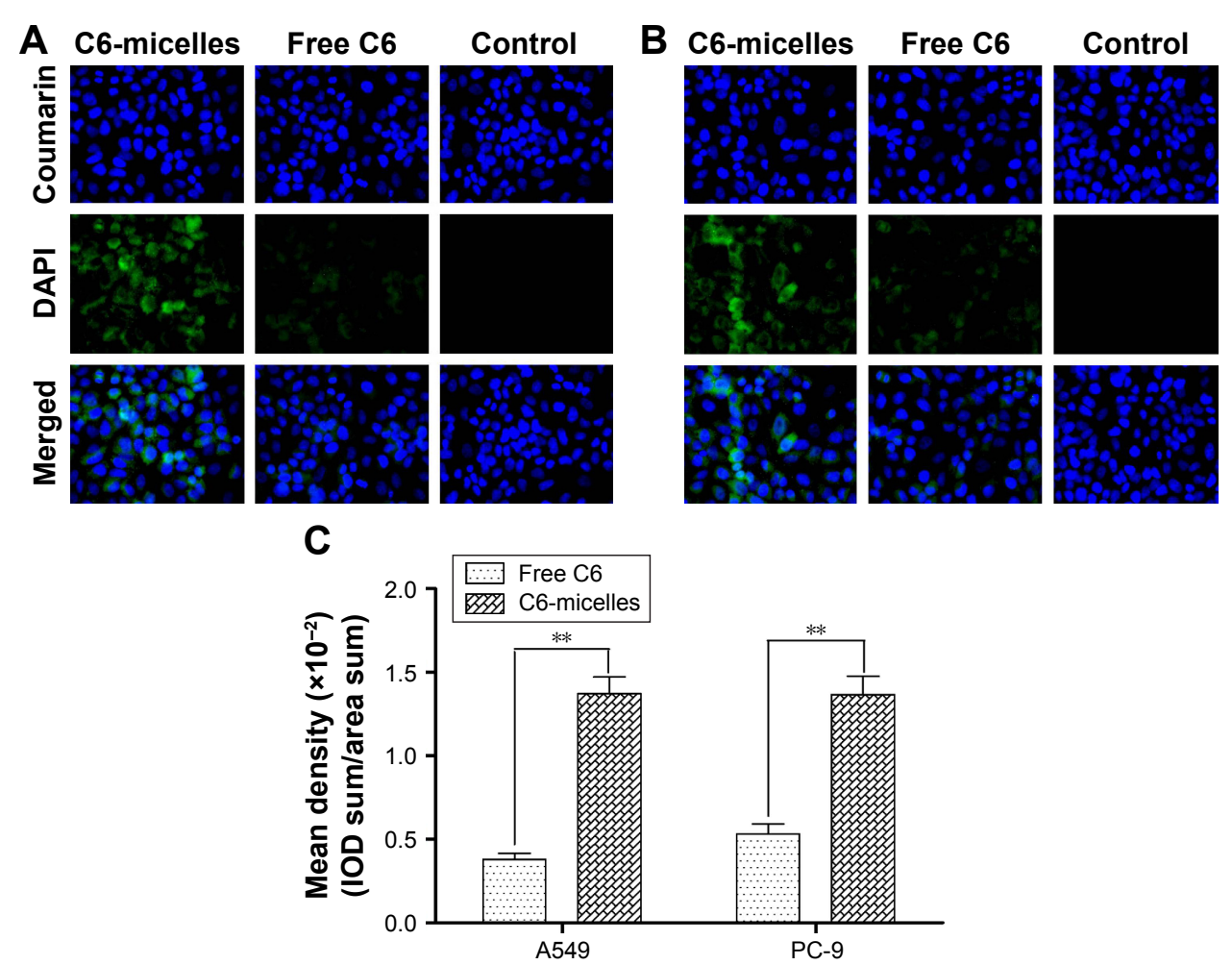

Figure 6 Cellular uptake of C6-micelles.

Notes: Images of A549 (A) and PC-9 (B) lung cancer cells treated with C6-micelles and free C6 (magnification 400x). (C) Mean values of integral optical density after treatment with $\mathrm{C} 6$-micelles and free $\mathrm{C} 6 . \mathrm{n}=3$, $* * \mathrm{P}<0.01$.

Abbreviations: C6, coumarin 6; DAPI, 4',6-diamidino-2-phenylindole; IOD, integrated optical density.

A

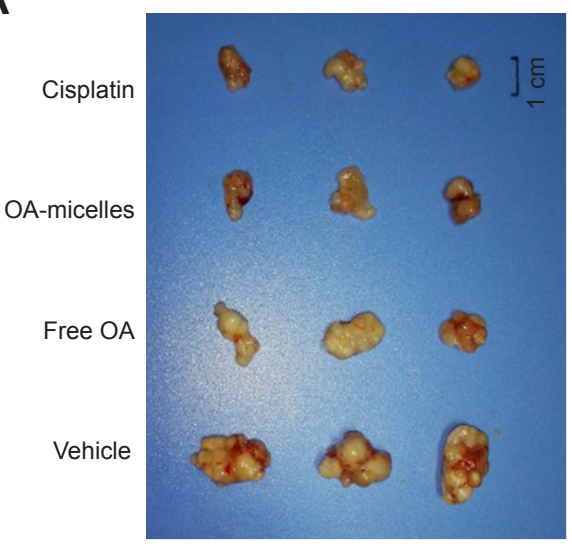

C

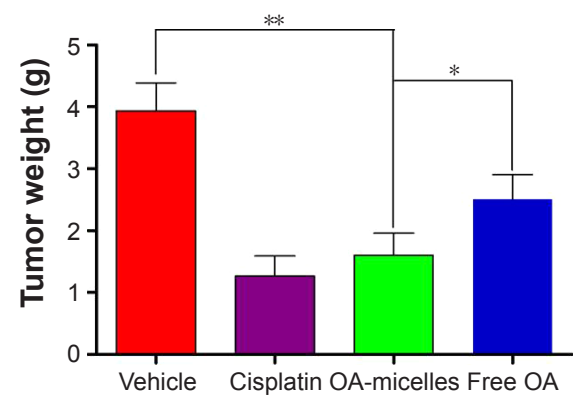

B

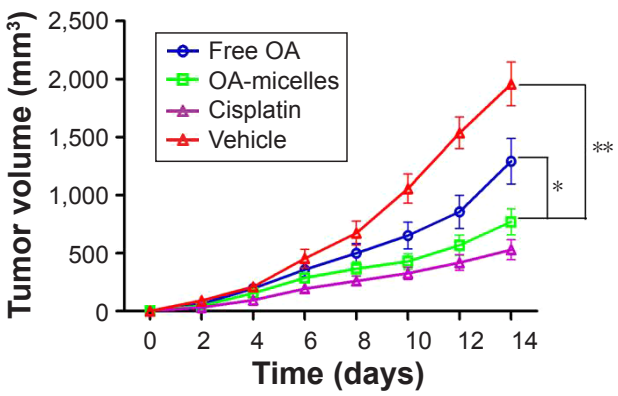

D

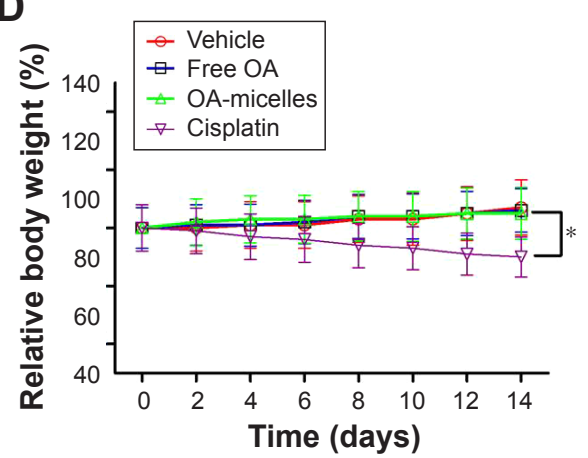

Figure 7 The antitumor effect of OA-micelles on mice bearing A549 cells.

Notes: (A) Xenograft tumors were excised on day 14 after initial treatment; (B) tumor volume changes as a function of time after intravenous injection of vehicle, free OA, OA-micelles, and cisplatin; (C) average tumor weight after last treatment; and (D) body weight changes of tumor-bearing mice after treatment. $* P<0.05$, $* * P<0.01$. Abbreviation: OA, oleanolic acid. 

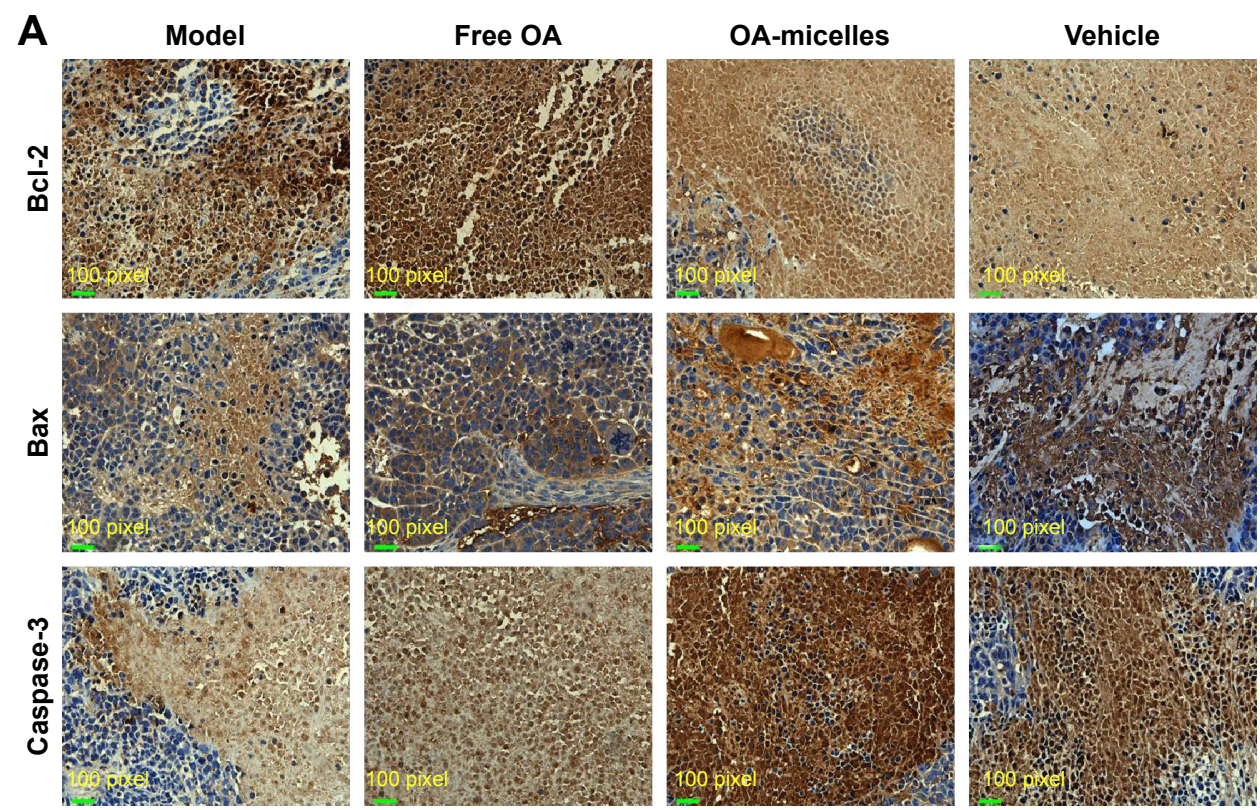

B

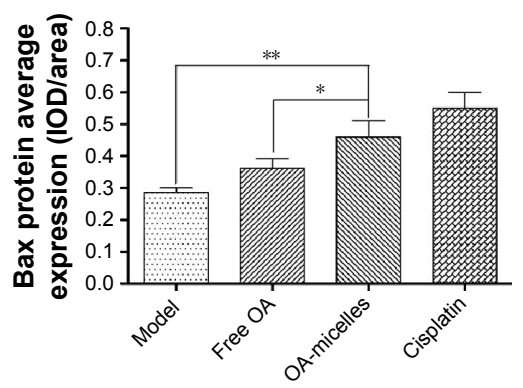

C

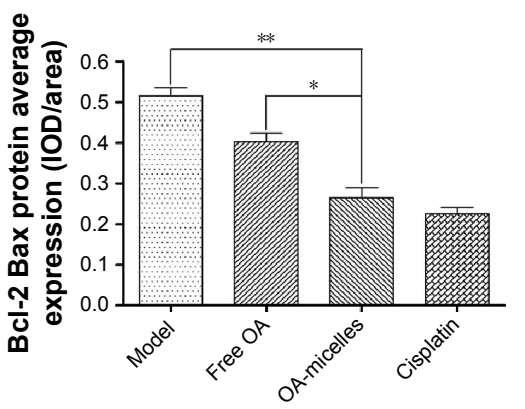

D

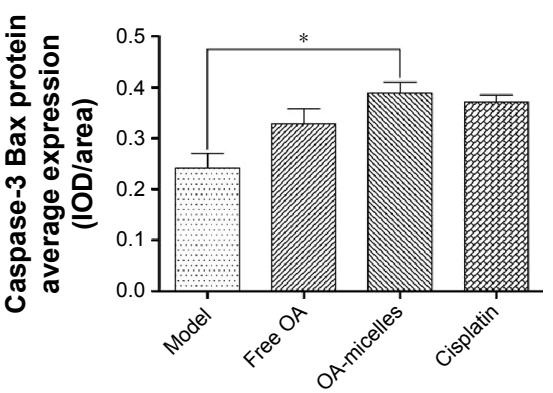

Figure 8 Immunohistochemistry for Bax, Bcl-2, and Caspase-3 from A549 xenografts.

Notes: Data expressed as mean $\pm \mathrm{SD}$. $\mathrm{n}=5$ or more. ${ }^{*} \mathrm{P}<0.05$, $* * \mathrm{P}<0.01$. Magnification $400 \times$. (A) Immunohistochemistry for Bax, Bcl-2, Caspase-3 in each group. (B) Expression of Bax protein in each group. (C) Expression of Bcl-2 protein in each group. (D) Expression of Caspase-3 protein in each group.

Abbreviations: IOD, integrated optical density; SD, standard deviation; OA, oleanolic acid.

that OA-micelles displayed a greater anticancer effect than free $\mathrm{OA}$ at the same concentration in vitro. The $\mathrm{IC}_{50}$ of OA-micelles was $20.9 \pm 3.7$ and $56.7 \pm 4.7 \mu \mathrm{M}$ for A549 and PC-9 cells, respectively, and the $\mathrm{IC}_{50}$ of free $\mathrm{OA}$ was $36.8 \pm 4.8$ and $82.7 \pm 7.8 \mu \mathrm{M}$ for A549 and PC-9 cells, respectively (Figure 9).

Annexin V-FITC and PI staining assay is a way to detect the apoptotic level of cells. ${ }^{40}$ Previously, OA was reported to induce apoptosis in various cell lines. ${ }^{41-43} \mathrm{Here}$, a choice was made to use the $\mathrm{IC}_{50}$ of free OA for A549 and PC-9 cells (36.8 and $82.7 \mu \mathrm{M}$, respectively). OA-micelles at the equivalent drug concentration were used. After $24 \mathrm{~h}$, the percentage of apoptotic A549 and PC-9 cells treated with OA-micelles was $67.67 \%$ and $71.37 \%$, respectively (Figure 9C). A higher percentage of apoptotic cells were observed in wells treated with OA-micelles than those treated with free OA (Figure 9C). These results indicate that the formation of micelles could enhance the cytotoxicity of the free OA.

\section{OA-micelles inhibited NSCLC cell migration and invasion}

To investigate the effect of free $\mathrm{OA}$ and OA-micelles in NSCLC metastasis, cell migration and invasion were examined by wound healing and transwell invasion assays, respectively. In the following experiments, 15 and $30 \mu \mathrm{M}$ of OA-micelles (measured as OA concentration) were applied to A549 and PC-9 cells, respectively. The wounds of NSCLC cells treated with free OA and OA-micelles failed to close after $24 \mathrm{~h}$ (Figure 10). The results showed that both OAmicelles and free OA inhibited migration of NSCLC cells. OA-micelles showed stronger migration inhibition than free OA $(P<0.05)$ in both A549 and PC-9 cells. The anti-invasive capacity of OA-micelles was also greater than that of free $\mathrm{OA}$ at the same concentration, as observed in a transwell assay (Figure 10E-H). 

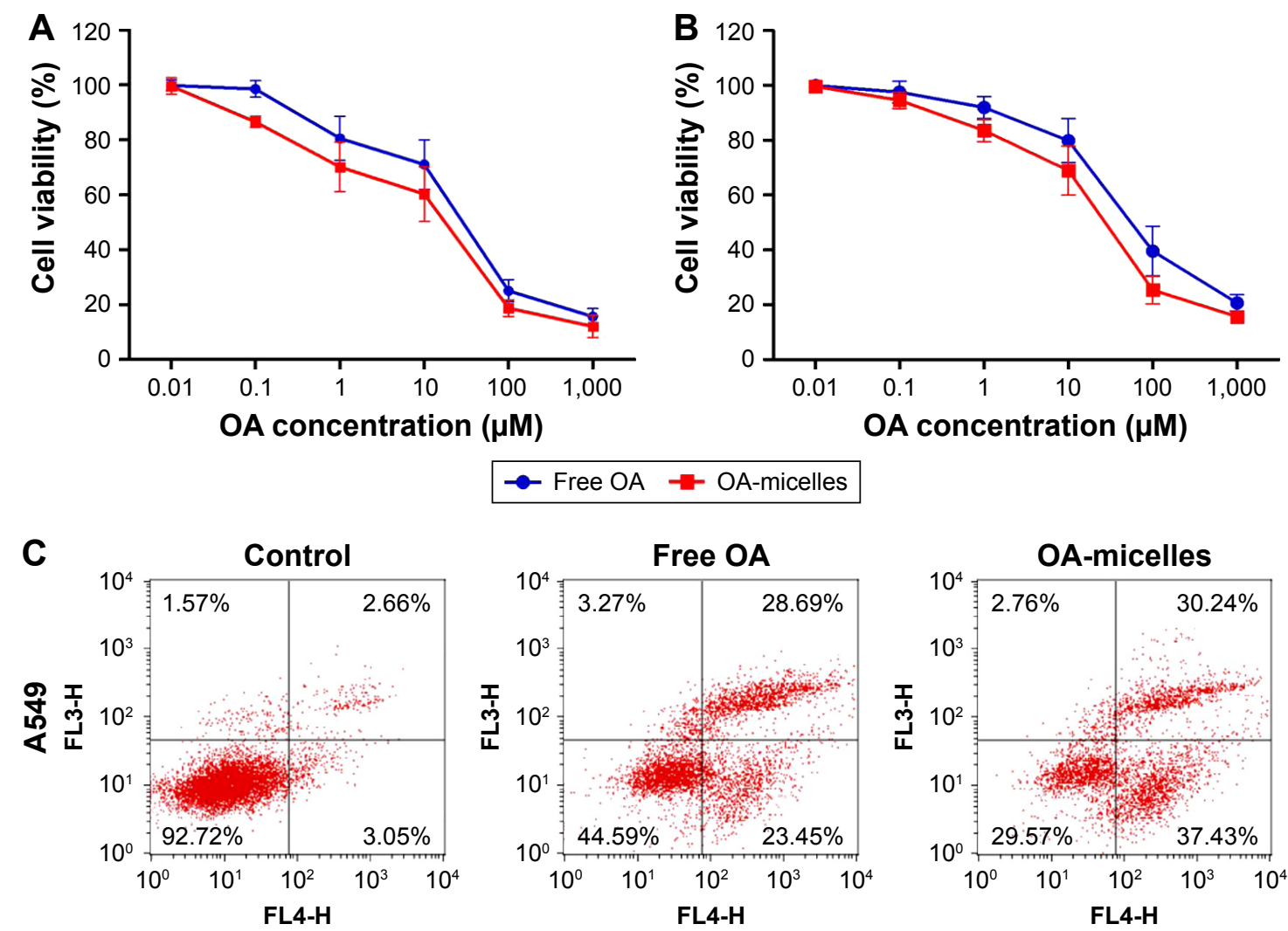

$\rightarrow$ Free OA $\rightarrow$ OA-micelles
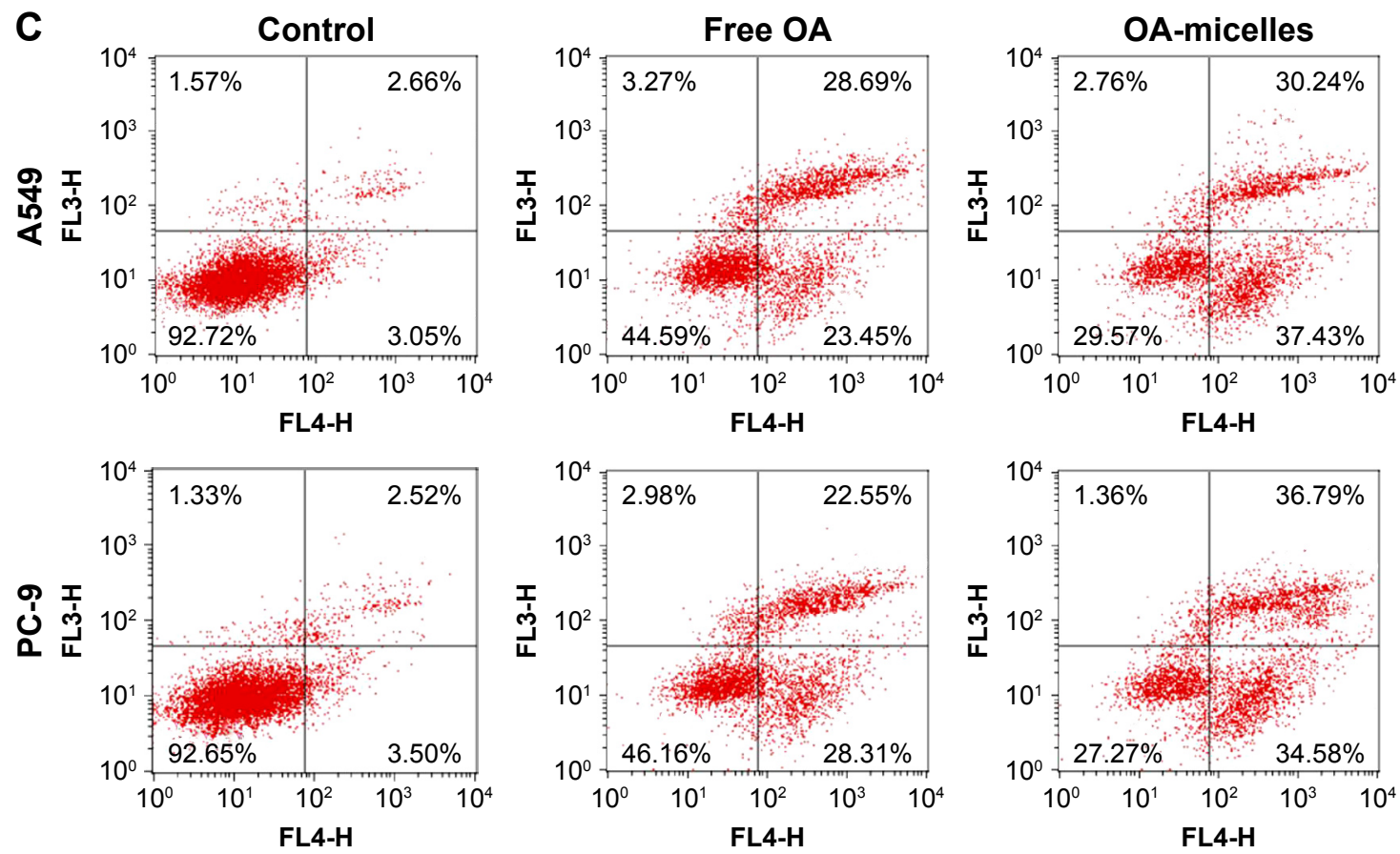

Figure 9 The in vitro antitumor activity of free $O A$ and $O A$-micelles.

Notes: MTT assay of A549 (A) and PC-9 (B) cells; (C) flow cytometry of Annexin V and PI staining. The cells were incubated with free OA and OA-micelles for 24 h. Data are presented as mean \pm standard deviation $(n=3)$.

Abbreviations: OA, oleanolic acid; MTT, 3-(4,5-dimethylthiazol-2-yl)-2,5-diphenyltetrazolium bromide; PI, propidium iodide.

\section{OA-micelles inhibited EMT of NSCLC cells}

The EMT is a pivotal event in tumor progression during which cancer cells undergo dramatic changes and acquire highly invasive properties. Immunofluorescence microscopy was used to explore the effects of OA-micelles on the expression of E-cadherin and N-cadherin in NSCLC cells. Treatment with OA-micelles increased the epithelial marker, E-cadherin, and decreased the mesenchymal marker, N-cadherin, in both cell lines (Figure 11). Similar results were obtained from Western blot analysis. To uncover the molecular mechanism of action of free OA and OA-micelles, the expression levels of Caspase-3, ERK, and p-ERK in NSCLC cells were determined. After $24 \mathrm{~h}$ exposure to free OA and OA-micelles, the levels of Caspase-3 and p-ERK increased, without any significant change in total ERK levels. The increase in Caspase-3 and p-ERK was not as great in the free OA group compared with that in the OA-micelles group (Figure 12). Furthermore, the ratio of $\mathrm{p}$-ERK/ERK increased considerably in the OAmicelles group. A549 and PC-9 cells showed the same trend. These data suggested an important role for ERK signaling in the anti-metastasis effect of OA-micelles.

\section{Discussion}

Natural products, including paclitaxel, have been used in the clinical treatment of various cancer types. ${ }^{44,45} \mathrm{OA}$ has also drawn extensive attention owing to its biological 

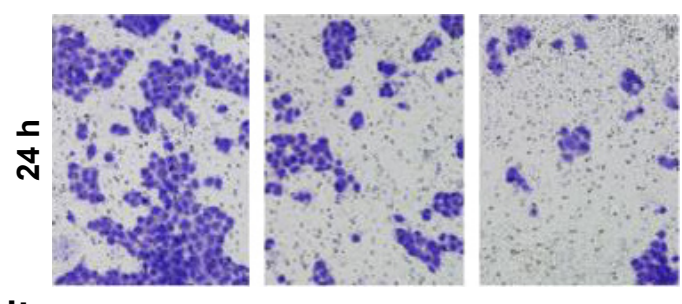

丩
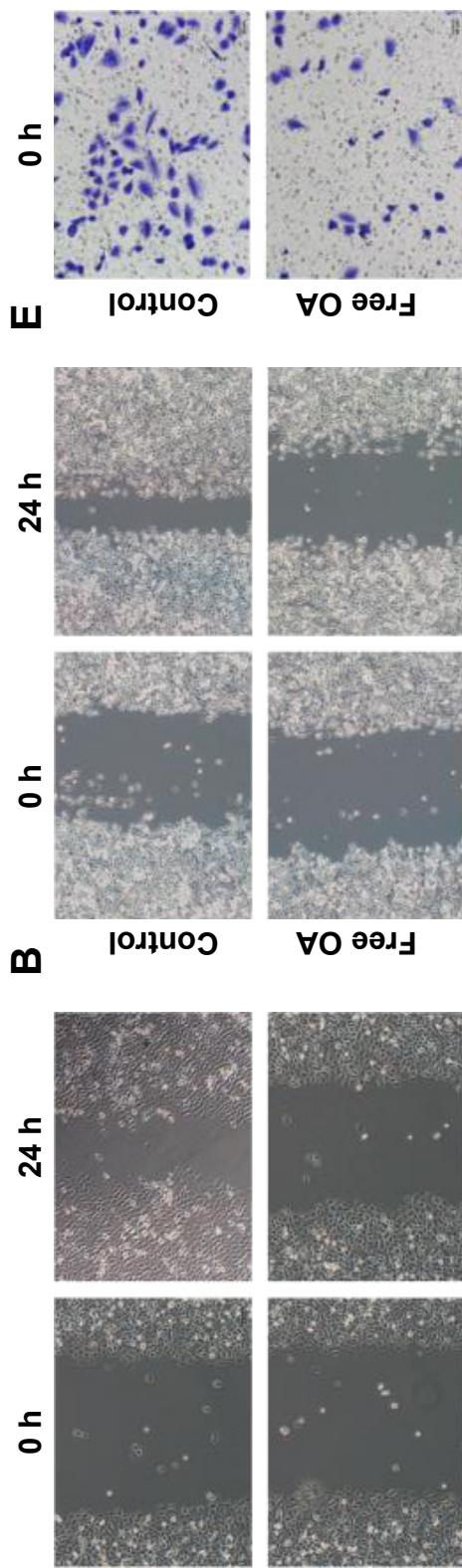

$<$

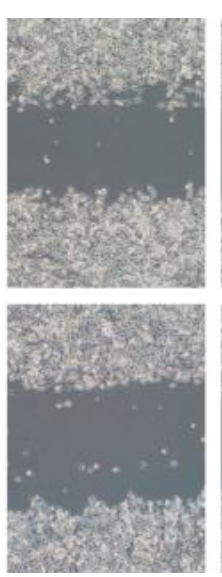

$\forall 0$ әәנ
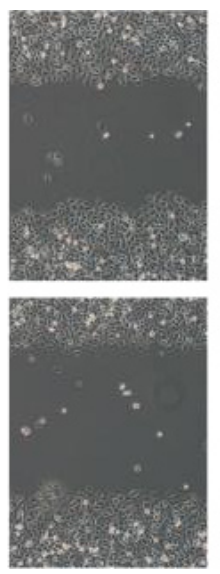

$\forall 0$ әәコ
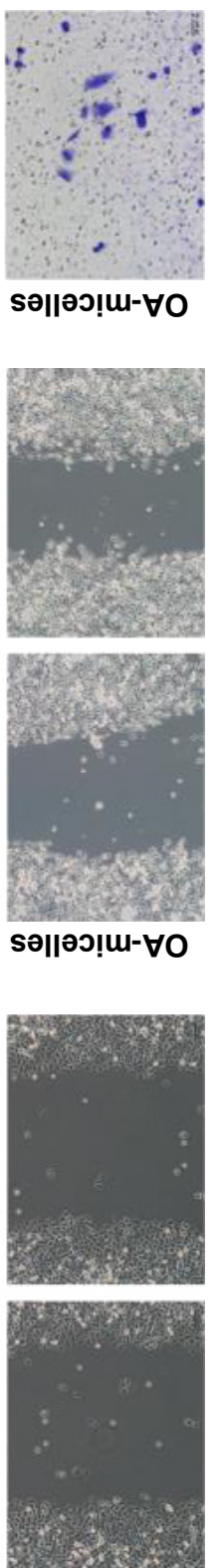

sə||əગ!ய- $\forall 0$

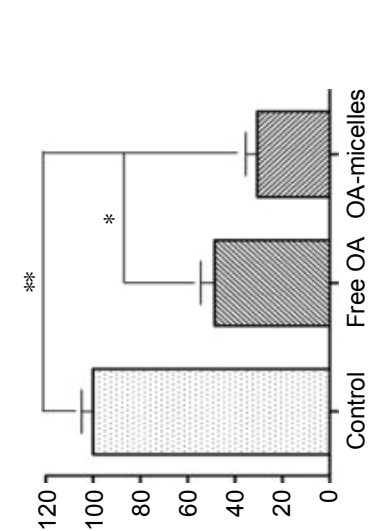

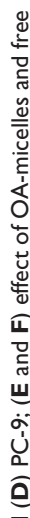

I (\%) s||әо әм!se^u|

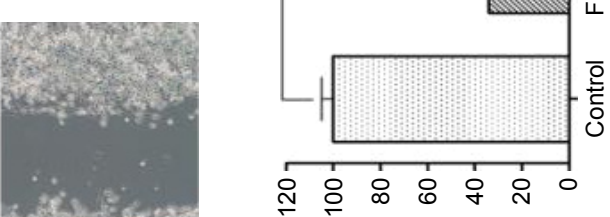

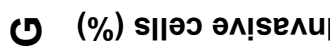

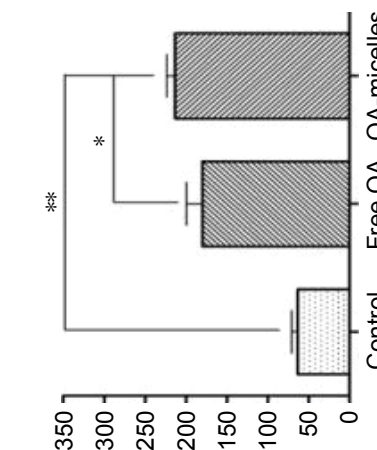

口 (url) чұбuә deэ

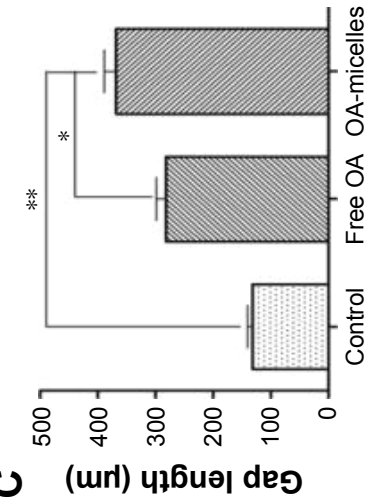

产

突

o

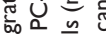

है

然守

致

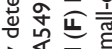

矛余栗

क्ष पे पे

夢出讨

กิิ

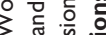

$0 \leqslant$ 늘

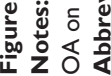




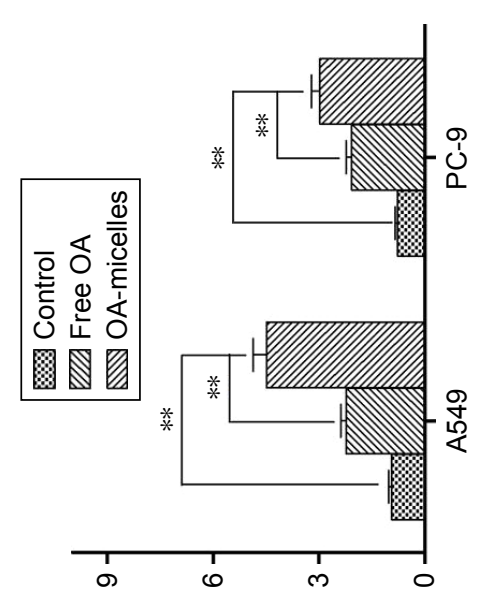

(uns eəje/uns gol)
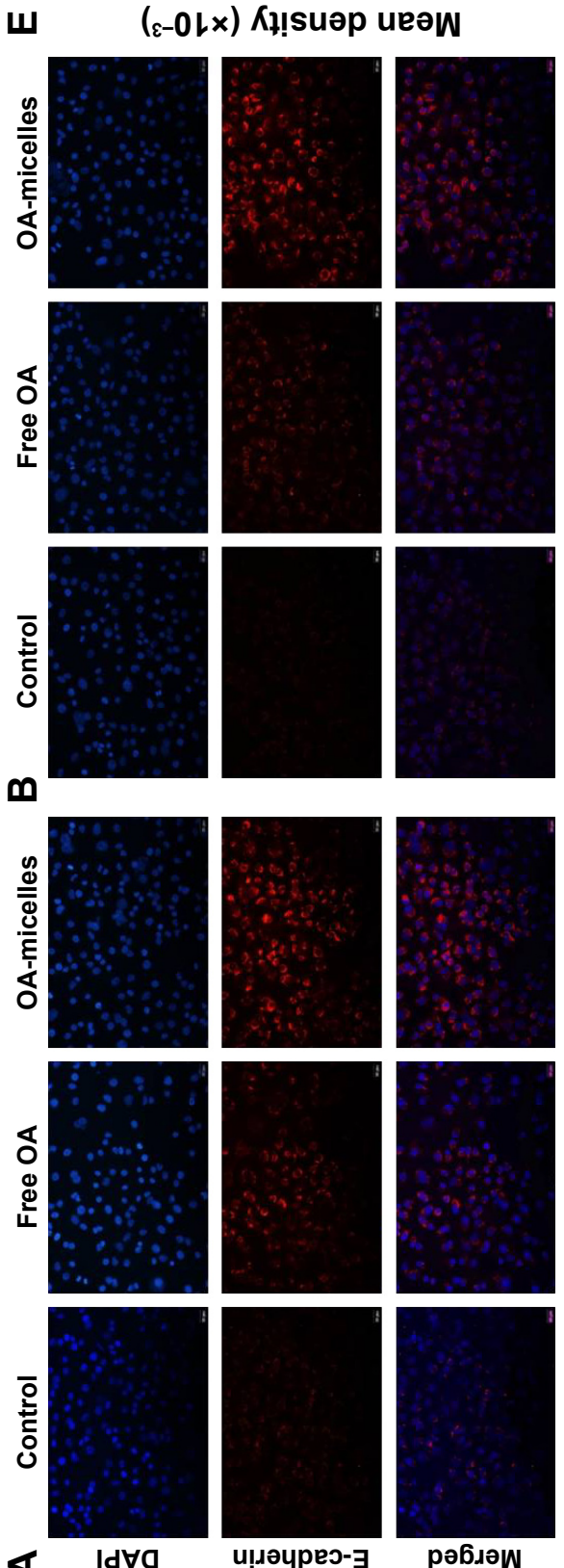

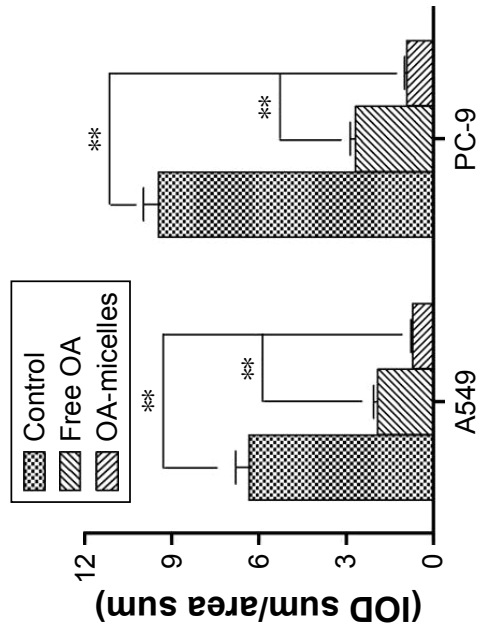

ᄂ $\quad(\varepsilon-0 l x) \Lambda_{\imath} !$ suəp ueəW
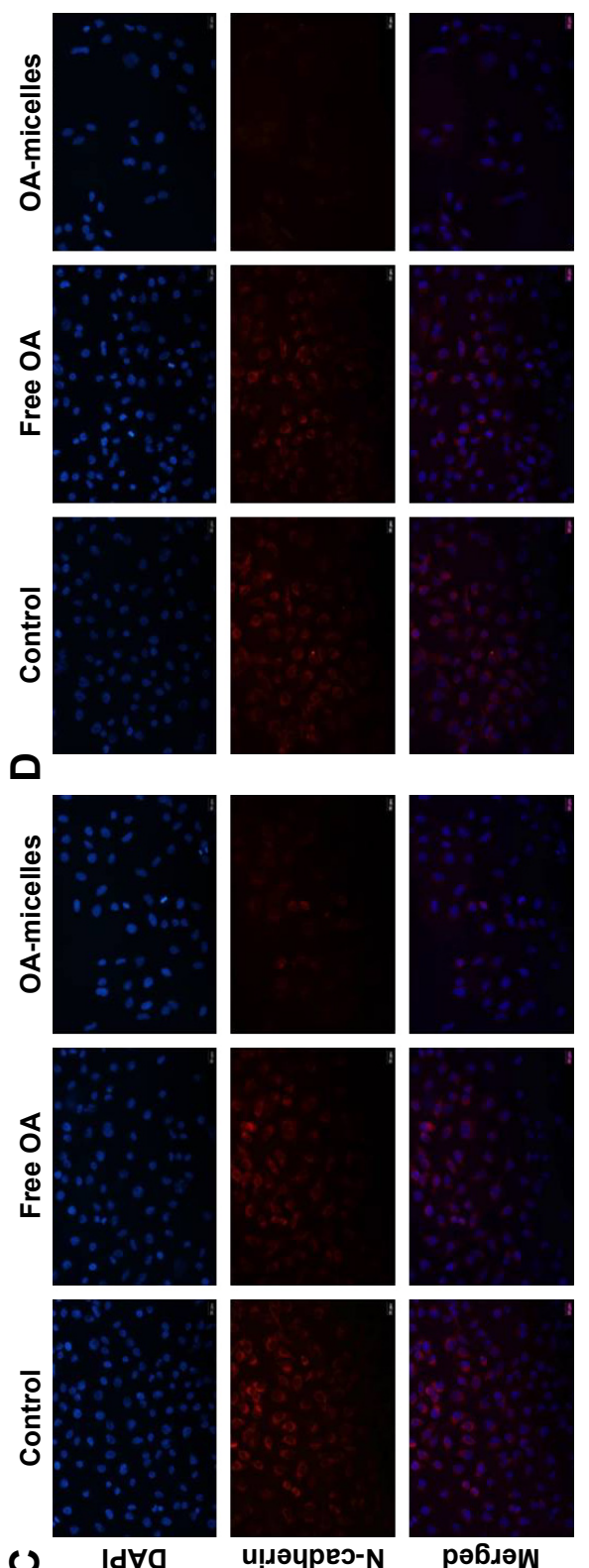

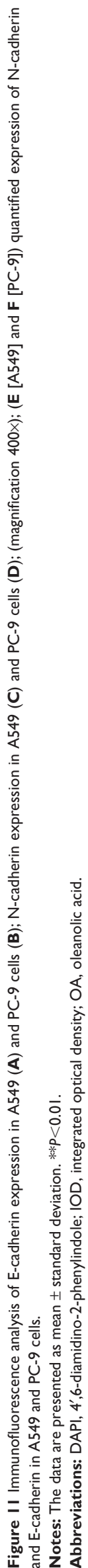


A

A549

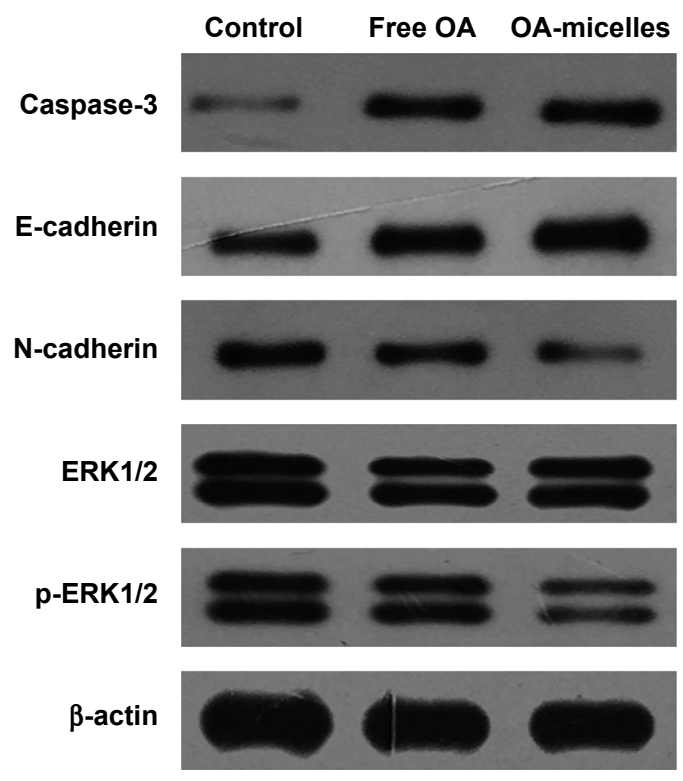

C

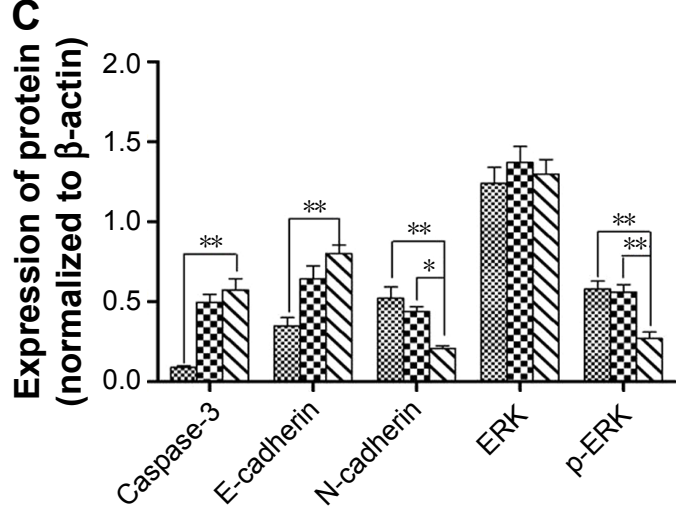

Control 8 Free OA $\triangle \square$ OA-micelles
B

PC-9
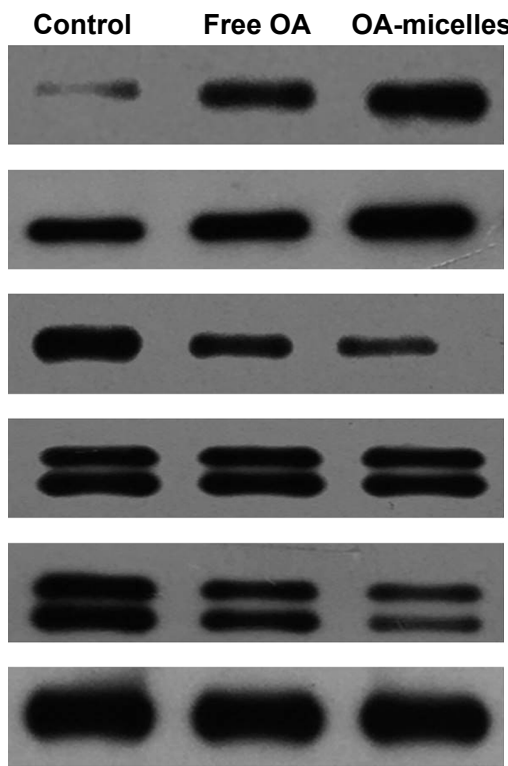

D

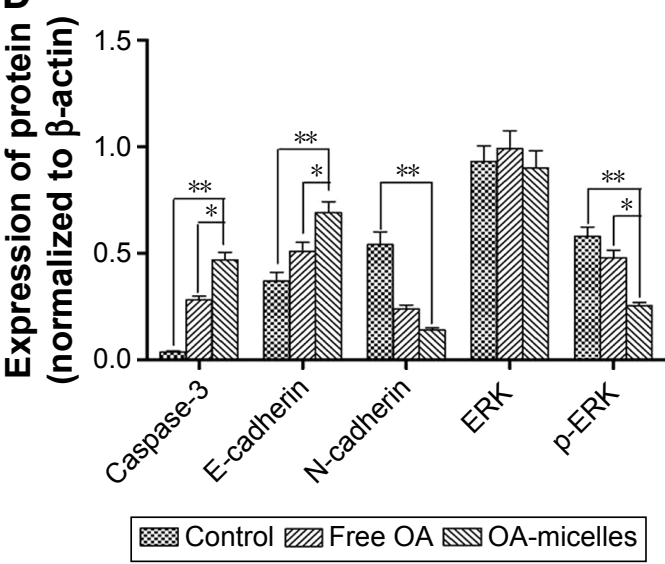

Figure 12 Western blot analysis of NSCLC cells treated with control media, free OA, and OA-micelles.

Notes: (A and B) Western blot analysis of the EMT-related proteins, Caspase-3 and p-ERK, in A549 and PC-9 cells. $\beta$-actin expression is shown as a loading control. (C [A549] and $\mathbf{D}[\mathrm{PC}-9]$ ) The figures are representative of three independent experiments. The data are presented as mean \pm standard deviation. $* P<0.05$, $* * P<0.01$.

Abbreviations: NSCLC, non-small-cell lung cancer; OA, oleanolic acid; EMT, epithelial-mesenchymal transition.

activity in cancer therapy. Unfortunately, the poor water solubility, low permeability, and significant efflux of OA have restricted its clinical use. ${ }^{46}$ Recently, polymer micelles and liposomes have been used as carriers in drug delivery to overcome the shortcomings of drugs with low solubility. An ideal carrier material for injection should be equipped with characteristics that improve the scatter and stability of drugs, enhance biological utilization, and accelerate the absorbance of drugs. Additionally, inhibiting P-glycoprotein (P-gp) efflux is a key property for solubilizers. ${ }^{47-49}$ TPGS possesses a lipophilic nonpolar head and a hydrophilic tail and would be an ideal surface-active agent to provide a high drug emulsification effect. ${ }^{50,51}$ It has been reported that a self-assembled mixture of micelles (TPGS-Icariside IIphospholipid complex) successfully improved the solubility of Icariside II and alleviated efflux in Caco-2 cells. ${ }^{10}$ As a pluronic excipient, P105 is a well-known polymeric inhibitor of P-gp and functions via inhibiting ATPase and fluidizing membrane. ${ }^{52,53}$ PEG/P105 micelles encapsulating doxorubicin could promote the radiosensitivity of doxorubicin-resistant tumor cells. ${ }^{54} \mathrm{P} 105$ is a $\mathrm{PEO}_{37}-$ $\mathrm{PPO}_{56}-\mathrm{PEO}_{37}$ triblock copolymer with impressive tumorsensitizing capacity and can be a favorable component of the micelles. Furthermore, Pluronic interaction promoted the accumulation of mitochondrial reactive oxygen species and cytochrome $\mathrm{C}$ in tumor cells, inducing apoptosis. ${ }^{55}$ Enhanced absorbability and reduced efflux greatly improved the antitumor efficiency of OA-micelles. Meanwhile, drug accumulation and retention in tumors may be another means of improving antitumor efficiency, as demonstrated in the 
cellular uptake assays and in vivo imaging of DiR-loaded micelles. The combined effect of the pluronic block polymer and passively targeted drug-resistant tumors by block copolymers may be the primary reason for the inhibition of tumor growth in the OA-micelles treatment groups.

In a successful drug delivery system, the rate at which the drug is released from preparations is important. Analysis of OA release using a dialysis system showed that $\sim 80 \%$ of OA was released from the free OA treatment after $24 \mathrm{~h}$, while $\sim 40 \%$ was released from the OA-micelle treatment over the same time period. Although OA cannot be dissolved in pure water, about half of OA in the OA-micelles was released after $48 \mathrm{~h}$, suggesting that OA could freely diffuse through the dialysis membrane. This might be due to the crystallization of free OA as a separate phase inside the micelle core, leading to a slower drug release. Moreover, slow release can reduce damage to the organ responsible for the metabolism of the drug, leading to fewer side effects.

Lung cancer is one of the leading causes of cancer-related deaths worldwide. Apoptosis, also known as programmed cell death, is a physical phenomenon that eliminates unnecessary and cancerous cells. Inducing apoptosis is the most widely used method used to treat cancer currently. The ability of internal or external stimuli to induce apoptosis is of immense therapeutic potential. This study demonstrated that OA inhibits tumor growth by inducing apoptosis. Another approach used to treat cancer is to inhibit tumor cell invasion. This study clearly shows that there is a significant difference in the tumor volume and weight after treatment with OAmicelles, and free OA, and the control treatment. Bcl-2, Bax, and Caspase- 3 play critical roles in apoptosis. ${ }^{56}$ Interestingly, Bcl-2 and Bax are generally detected at the same time in the progression of apoptosis, and the ratio of $\mathrm{Bcl}-2 / \mathrm{Bax}$ reflects the degree of apoptosis. Bax and Caspase-3 are pro-apoptotic proteins and $\mathrm{Bcl}-2$ plays an anti-apoptotic role. In nude mice, the pro-apoptotic indicators, Caspase-3 and Bax, increased after administration of OA-micelles. In vitro experiments also revealed that the rate of apoptosis in NSCLC cells treated with OA-micelles was significantly higher than that of cells treated with the same concentration of free OA. Western blot analysis showed a significant increase in Caspase- 3 and the $\mathrm{Bax} / \mathrm{Bcl}-2$ ratio after $24 \mathrm{~h}$ exposure to OA-micelles but not after $24 \mathrm{~h}$ exposure to free OA or in the control.

The migration and invasion abilities of cancer cells are major causes of disease progression in patients. In this study, wound healing and transwell invasion assays were used to study the anti-metastasis effects of OA-micelles on NSCLC cells. A stronger inhibitory effect on cell migration and invasion was observed in cells treated with OA-micelles compared with those subjected to free OA and control treatments. The EMT plays an important role in the invasion and metastasis of tumors. ${ }^{57}$ The process is characterized by the loss of polarity and connectivity between epithelial cells and the acquisition of an interstitial cell phenotype under the influence of numerous factors. ${ }^{58}$ During this process, mesenchymal markers such as $\mathrm{N}$-cadherin are upregulated, and adhesion molecules such as E-cadherin are downregulated. Western blotting and immunofluorescence analyses were used to determine the expression levels of E-cadherin and N-cadherin. The results showed that the expression of E-cadherin was significantly increased and $\mathrm{N}$-cadherin was decreased in the OA-micelles group, inferring that OAmicelles can affect the metastasis of NSCLC.

\section{Conclusion}

In this study, polymeric micelles loaded with OA were prepared and characterized. OA-micelles displayed suitable particle size, zeta potential, and in vitro release. In vivo images indicated benign passive accumulation of micelles in tumor tissues. The micelles can achieve better inhibition of cell growth in vivo and have a stronger apoptosis-inducing effect in NSCLC in vitro than free OA. Cell cytotoxicity experiments and wound healing and transwell assays showed increased antitumor efficacy of OA-micelles than free OA in vitro. Western blot and immunofluorescence analyses demonstrated that the degree of apoptosis in the micelles group was higher than that of free OA. In conclusion, OA-micelles displayed higher antitumor efficacy than free OA in both A549 and PC-9 cells. Construction of OAmicelles with TPGS/P105 can significantly enhance the OA sensitivity of NSCLC and provide an effective method for the treatment of lung cancer. Therefore, the micelles could be used as a drug delivery carrier to improve the therapeutic effects of OA. In addition, TPGS/P105 micelles may also have the potential to increase the bioavailability of drugs with poor solubility.

\section{Acknowledgments}

This work was supported by the National Natural Science Foundation of China (number 81303275); Traditional Chinese Medicine for Public Interest Research from Ministry of Finance of China (number 201507004); Natural Science Foundation of Jiangsu (number BK20141507, People's Republic of China), and Anhui universities Provincial Natural Science Key Research Project (number KJ2014A242, People's Republic of China). 


\section{Disclosure}

The authors report no conflicts of interest in this work.

\section{References}

1. Khan M, Khan M, Almarri AH, et al. Apoptosis inducing ability of silver decorated highly reduced graphene oxide nanocomposites in A549 lung cancer. Int J Nanomedicine. 2016;11:873-883.

2. Song J, Feng L, Zhong RL, et al. Icariside II inhibits the EMT of NSCLC cells in inflammatory microenvironment via down-regulation of Akt/ NF-kB signaling pathway. Mol Carcinog. Epub Feb 92016.

3. Li HF, Wang XA, Xiang SS, et al. Oleanolic acid induces mitochondrialdependent apoptosis and $\mathrm{G} 0 / \mathrm{G} 1$ phase arrest in gallbladder cancer cells. Drug Des Devel Ther. 2015;9(30):17-30.

4. Li X, Song Y, Peng Z, et al. Oleanolic acid inhibits cell survival and proliferation of prostate cancer cells in vitro and in vivo through the PI3K/Akt pathway. Tumour Biol. 2016;37(6):7599-7613.

5. Nelson AT, Camelio AM, Claussen KR, et al. Synthesis of oxygenated oleanolic and ursolic acid derivatives with anti-inflammatory properties. Bioorg Med Chem Lett. 2015;25(19):4342-4346.

6. Greatrex BW, Daines AM, Hook S, et al. Synthesis, formulation, and adjuvanticity of monodesmosidic saponins with olenanolic acid, hederagenin and gypsogenin aglycones, and some $\mathrm{C}-28$ ester derivatives. ChemistryOpen. 2015;4(6):740-755.

7. Xu G, Yu X, Zhang J, et al. Robust aptamer-polydopamine-functionalized M-PLGA-TPGS nanoparticles for targeted delivery of docetaxel and enhanced cervical cancer therapy. Int J Nanomedicine. 2016;11: 2953-2965.

8. Caiolfa VR, Zamai M, Fiorino A, et al. Polymer-bound camptothecin: initial biodistribution and antitumour activity studies. J Control Release. 2000;65(1-2):105-119.

9. Chen L, Sha X, Jiang X, Chen Y, Ren Q, Fang X. Pluronic P105/F127 mixed micelles for the delivery of docetaxel against Taxol-resistant non-small cell lung cancer: optimization and in vitro, in vivo evaluation. Int J Nanomedicine. 2013;8(10):73-84.

10. Song J, Huang H, Xia Z, et al. TPGS/phospholipids mixed micelles for delivery of icariside II to multidrug-resistant breast cancer. Integr Cancer Ther. 2016;15(3):390-399.

11. Hou J, Wang J, Sun E, et al. Preparation and evaluation of icariside IIloaded binary mixed micelles using Solutol ${ }^{\circledR}$ HS15 and Pluronic F127 as carriers. Drug Deliv. 2016;11:1-24.

12. Yan HM, Song J, Zhang ZH, Jia XB. Optimization and anticancer activity in vitro and in vivo of baohuoside I incorporated into mixed micelles based on lecithin and Solutol HS 15. Drug Deliv. 2016;23(8): 2911-2918.

13. Mebarek N, Aubert-Pouëssel A, Gérardin C, Vicente R, Devoisselle JM, Bégu S. Polymeric micelles based on poly(methacrylic acid) blockcontaining copolymers with different membrane destabilizing properties for cellular drug delivery. Int J Pharm. 2013;454(2):611-620.

14. Zheng S, Xiang G, Liu X, et al. Biodegradable micelles enhance the antiglioma activity of curcumin in vitro and in vivo. Int J Nanomedicine. 2016;11:2721-2736.

15. Cai S, Shi CH, Zhang X, et al. Self-microemulsifying drug-delivery system for improved oral bioavailability of 20(S)-25-methoxyldammarane-3 $\beta, 12 \beta$, 20-triol: preparation and evaluation. Int J Nanomedicine. 2013;9(9):913-920.

16. Song J, Zhong R, Huang H, et al. Combined treatment with Epimedium koreanum Nakai extract and gefitinib overcomes drug resistance caused by T790M mutation in non-small cell lung cancer cells. Nutr Cancer. 2014; 66(4):1-8.

17. Wang CF, Yuan JR, Qin D, et al. Protection of tauroursodeoxycholic acid on high glucose-induced human retinal microvascular endothelial cells dysfunction and streptozotocin-induced diabetic retinopathy rats. J Ethnopharmacol. 2016;185:162-170.

18. Sun M, Yin W, Dong X, Yang W, Zhao Y, Yin M. Fluorescent supramolecular micelles for imaging-guided cancer therapy. Nanoscale. 2016; 8(9):5302-5312.
19. Chen W, Meng F, Cheng R, Deng C, Feijen J, Zhong Z. Facile construction of dual-bioresponsive biodegradable micelles with superior extracellular stability and activated intracellular drug release. $J$ Control Release. 2015;210:125-133.

20. Cheng X, Yan H, Jia X, Zhang Z. Preparation and in vivo/in vitro evaluation of formononetin phospholipid/vitamin E TPGS micelles. J Drug Target. 2016;24(2):1-8.

21. Liu MJ, Qu D, Chen Y, Liu CY, Liu YP, Ding XF. Preparation of novel butyryl galactose ester-modified coix component microemulsions and evaluation on hepatoma-targeting in vitro and in vivo. Drug Deliv. Epub 2016 Jun 10:1-23.

22. Schaal JL, Li X, Mastria E, et al. Injectable polypeptide micelles that form radiation crosslinked hydrogels in situ for intratumoral radiotherapy. J Control Release. 2016;228:58-66.

23. Wang K, Zhang T, Liu L, et al. Novel micelle formulation of curcumin for enhancing antitumor activity and inhibiting colorectal cancer stem cells. Int J Nanomedicine. 2011;7(17):4487-4497.

24. Bosch RV, Alvarez-Flores MP, Maria DA, Chudzinski-Tavassi AM. Hemolin triggers cell survival on fibroblasts in response to serum deprivation by inhibition of apoptosis. Biomed Pharmacother. 2016;82: 537-546.

25. Awasthi BP, Kathuria M, Pant G, Kumari N, Mitra K. Plumbagin, a plant-derived naphthoquinone metabolite induces mitochondria mediated apoptosis-like cell death in Leishmania donovani: an ultrastructural and physiological study. Apoptosis. 2016;21(8):1-13.

26. Amna T, Hassan MS, Yang J, et al. Virgin olive oil blended polyurethane micro/nanofibers ornamented with copper oxide nanocrystals for biomedical applications. Int J Nanomedicine. 2014;9(1): 891-898.

27. You W, Liu T, He L. Enhancement of triptolide-loaded micelles on tumorigenicity inhibition of human ovarian cancer. J Biomater Sci Polym Ed. 2016;27(7):1-21.

28. Xu W, Burke JF, Pilla S, Chen H, Jaskula-Sztul R, Gong S. Octreotidefunctionalized and resveratrol-loaded unimolecular micelles for targeted neuroendocrine cancer therapy. Nanoscale. 2013;5(20):9924-9933.

29. Talelli M, Rijcken CJF, Oliveira S, et al. Nanobody-shell functionalized thermosensitive core-crosslinked polymeric micelles for active drug targeting. J Control Release. 2011;153(1):93-102.

30. Savić R, Azzam T, Eisenberg A, Nedev H, Rosenberg L, Maysinger D. Block-copolymer micelles as carriers of cell signaling modulators for the inhibition of JNK in human islets of Langerhans. Biomaterials. 2009;30(21):3597-3604.

31. Talelli M, Rijcken CJF, Oliveira S, et al. Reprint of "nanobody - shell functionalized thermosensitive core-crosslinked polymeric micelles for active drug targeting". J Control Release. 2011;153(1):93-102.

32. Gurunathan S, Park JH, Han JW, Kim JH. Comparative assessment of the apoptotic potential of silver nanoparticles synthesized by Bacillus tequilensis and Calocybe indica in MDA-MB-231 human breast cancer cells: targeting p53 for anticancer therapy. Int J Nanomedicine. 2015;10(2):4203-4223.

33. Akiyama $Y$, Morikawa $T$, Maeda $D$, et al. Increased CXCR3 expression of infiltrating plasma cells in hunner type interstitial cystitis. Sci Rep. 2016;6:28652.

34. Hou J, Jia XB, Zhang ZH, et al. Improved oral bioavailability and anticancer efficacy on breast cancer of paclitaxel via Novel Soluplus $\left({ }^{\circledR}\right)$-Solutol $\left({ }^{\circledR}\right)$ HS15 binary mixed micelles system. Int J Pharm. 2016;512(1):186-193.

35. Lv L, Qiu K, Chen C, et al. Amphiphilic copolymeric micelles for doxorubicin and curcumin co-delivery to reverse multidrug resistance in breast cancer. J Biomed Nanotechnol. 2016;12(5): 973-985.

36. Golding CG, Lamboo LL, Beniac DR, Booth TF. The scanning electron microscope in microbiology and diagnosis of infectious disease. Sci Rep. 2016;23(6):1-8.

37. Taylor RM, Sillerud LO. Paclitaxel-loaded iron platinum stealth immunomicelles are potent MRI imaging agents that prevent prostate cancer growth in a PSMA-dependent manner. Int J Nanomedicine. 2012; 7(7):4341-4352. 
38. Wang J, Fang G, Meng Y, et al. Rapamycin/DiR loaded lipid-polyaniline nanoparticles for dual-modal imaging guided enhanced photothermal and antiangiogenic combination therapy. J Control Release. 2016;237:23-34.

39. Cho H, Indig GL, Weichert J, Shin HC, Kwon GS. In vivo cancer imaging by poly(ethylene glycol)-b-poly( $\varepsilon$-caprolactone) micelles containing a near-infrared probe. Nanomedicine . 2011;8(2):228-236.

40. Montazeri M, Sadeghizadeh M, Pilehvar-Soltanahmadi Y, et al. Dendrosomal curcumin nanoformulation modulate apoptosis-related genes and protein expression in hepatocarcinoma cell lines. Int J Pharm. 2016;509(1-2):244-254.

41. Liu J, Leina MA, Chen X, et al. ERK inhibition sensitizes cancer cells to oleanolic acid-induced apoptosis through ERK/Nrf2/ROS pathway. Tumor Biol. 2015;37(6):8181-8187.

42. Akl MR, Elsayed HE, Ebrahim HY, Haggag EG, Kamal AM, El Sayed KA. 3-O-[N-(p-fluorobenzenesulfonyl)-carbamoyl]-oleanolic acid, a semisynthetic analog of oleanolic acid, induces apoptosis in breast cancer cells. Eur J Pharmacol. 2014;740:209-217.

43. Liu J, Wu N, Ma LN, et al. p38 MAPK signaling mediates mitochondrial apoptosis in cancer cells induced by oleanolic acid. Asian Pac J Cancer Prev. 2014;15(15):4519-4525.

44. Ahmed A, Saleh ES, Mohamed A, et al. Rational design and synthesis of topoisomerase I and II inhibitors based on oleanolic acid moiety for new anti-cancer drugs. Bioorg Med Chem. 2013;22(1):211-220.

45. He Z, Wan X, Schulz A, et al. A high capacity polymeric micelle of paclitaxel: implication of high dose drug therapy to safety and in vivo anti-cancer activity. Biomaterials. 2016;101:296-309.

46. Jiang Q, Yang X, Du P, Zhang H, Zhang T. Dual strategies to improve oral bioavailability of oleanolic acid: enhancing water-solubility, permeability and inhibiting cytochrome P450 isozymes. Eur J Pharm Biopharm. 2016;99:65-72.

47. Dai L, Yu Y, Zhong L, et al. Photosensitizer enhanced disassembly of amphiphilic micelle for ROS-response targeted tumor therapy in vivo. Biomaterials. 2016;104:1-17.

48. Ke Z, Hou X, Jia X. Design and optimization of self-nanoemulsifying drug delivery systems for improved bioavailability of cyclovirobuxine D. Drug Des Devel Ther. 2016;28(10):2049-2060.
49. Hu Q, Rijcken CJF, Gaal EV, et al. Tailoring the physicochemical properties of core-crosslinked polymeric micelles for pharmaceutical applications. J Control Release. Epub 2016 Jul 9.

50. Sonali, Agrawal P, Singh RP, et al. Transferrin receptor-targeted vitamin E TPGS micelles for brain cancer therapy: preparation, characterization and brain distribution in rats. Drug Deliv. 2016;23(5): 1788-1798

51. Assanhou AG, Li W, Zhang L, et al. Reversal of multidrug resistance by co-delivery of paclitaxel and lonidamine using a TPGS and hyaluronic acid dual-functionalized liposome for cancer treatment. Biomaterials. 2015;73:284-295

52. Niu J, Wang A, Ke Z, Zheng Z. Glucose transporter and folic acid receptor-mediated Pluronic P105 polymeric micelles loaded with doxorubicin for brain tumor treating. J Drug Target. 2014;22(8):712-723.

53. Kar S, Ukil A, Das PK. Cystatin cures visceral leishmaniasis by NF- $\kappa \mathrm{B}$-mediated proinflammatory response through co-ordination of TLR/MyD88 signaling with p105-Tp12-ERK pathway. Eur J Immunol. 2011;41(1):116-127.

54. $\mathrm{Xu} \mathrm{W}$, Han M, Diao Y, et al. Doxorubicin encapsulated in micelles enhances radiosensitivity in doxorubicin-resistant tumor cells. Discov Med. 2014;18(99):169-177.

55. Alakhova DY, Rapoport NY, Batrakova EV, et al. Differential metabolic responses to pluronic in MDR and non-MDR cells: a novel pathway for chemosensitization of drug resistant cancers. J Control Release. 2010; 142(1):89-100.

56. You Q, Wu Z, Wu B, et al. Naringin protects cardiomyocytes against hyperglycemia-induced injuries in vitro and in vivo. J Endocrinol. 2016;230(2):197-214.

57. Ricciuti B, Mencaroni C, Paglialunga L, et al. Long noncoding RNAs: new insights into non-small cell lung cancer biology, diagnosis and therapy. Med Oncol. 2016;33(18):1-12.

58. Yang L, Bai HS, Deng Y, Fan L. High MALAT1 expression predicts a poor prognosis of cervical cancer and promotes cancer cell growth and invasion. Eur Rev Med Pharmacol Sci. 2015;19(17):3187-3193.
International Journal of Nanomedicine

\section{Publish your work in this journal}

The International Journal of Nanomedicine is an international, peerreviewed journal focusing on the application of nanotechnology in diagnostics, therapeutics, and drug delivery systems throughout the biomedical field. This journal is indexed on PubMed Central, MedLine, CAS, SciSearch ${ }^{\circledR}$, Current Contents ${ }^{\circledR} /$ Clinical Medicine,

\section{Dovepress}

Journal Citation Reports/Science Edition, EMBase, Scopus and the Elsevier Bibliographic databases. The manuscript management system is completely online and includes a very quick and fair peer-review system, which is all easy to use. Visit http://www.dovepress.com/ testimonials.php to read real quotes from published authors. 